\title{
BISMUT-ELWORTHY-LI FORMULA, SINGULAR SDES, FRACTIONAL BROWNIAN MOTION, MALLIAVIN CALCULUS, STOCHASTIC FLOWS, STOCHASTIC VOLATILITY*
}

\author{
OUSSAMA AMINE ${ }^{\dagger}$, EMMANUEL COFFIE $\ddagger$, FABIAN HARANG §, AND FRANK PROSKE
}

\begin{abstract}
In this paper we derive a Bismut-Elworthy-Li type formula with respect to strong solutions to singular stochastic differential equations (SDE's) with additive noise given by a multidimensional fractional Brownian motion with Hurst parameter $H<1 / 2$. "Singular" here means that the drift vector field of such equations is allowed to be merely bounded and integrable. As an application we use this representation formula for the study of the $\delta$ price sensitivity of financial claims based on a stock price model with stochastic volatility, whose dynamics is described by means of fractional Brownian motion driven SDE's.

Our approach for obtaining these results is based on Malliavin calculus and arguments of a recently developed "local time variational calculus".
\end{abstract}

Keywords. Bismut-Elworthy-Li formula, singular SDEs, fractional Brownian motion, Malliavin calculus, stochastic flows, stochastic volatility

AMS subject classifications. $60 \mathrm{H} 10 ; 49 \mathrm{~N} 60 ; 91 \mathrm{G} 80$

\section{Introduction}

In recent years the construction and computation of risk measures have become an indispensable tool for the risk analysis and risk management of portfolios in banks and insurance companies worldwide. An important class of risk measures often applied by investors on financial markets to hedge their positions is given by the "greeks". These are market sensitivities usually denoted by Greek letters e.g. "Delta", "Gamma", "Rho", "Theta", "Vega"..., and hence the name. For example the Delta $\Delta$, which can be used for the construction of delta hedges in portfolio management, measures the sensitivity of price changes of financial derivatives with respect to the initial price of the underlying asset. Roughly speaking, greeks are derivatives with respect to a parameter $\lambda$ of a (risk-neutral) price, that is, for example of the form

$$
\frac{\partial}{\partial \lambda} E\left[\Phi\left(\left(X_{T}^{\lambda}\right)\right)\right]
$$

where $\Phi$ is the payoff function of a claim and $X_{T}^{\lambda}$ the underlying asset at terminal time $T$, which depends on $\lambda$.

In general, greeks cannot be obtained by closed-form formulas, especially in the case of discontinuous payoff functions. Therefore, one has to resort to numerical techniques to approximate such sensitivities. A ground breaking method in this direction, which is also applicable to path-dependent options, has been developed in Fournié et al. [14], [15]. Assuming that the dynamics of asset prices $X_{t}=X_{t}^{\lambda}$ is modeled by a stochastic

\footnotetext{
*Received 17 Mar 2019, and accepted 13 Apr 2020.

${ }^{\dagger}$ Department of Mathematics, University of Oslo, P.O. Box 1053, Blindern, N-0316 Oslo, Norway, (oussamaa@math.uio.no).

${ }^{\ddagger}$ Department of Mathematics and Statistics, University of Strathclyde, Glasgow G1 1XH, UK, (emmanuel.coffie@strath.ac.uk).

$\S$ Department of Mathematics, University of Oslo, P.O. Box 1053, Blindern, N-0316 Oslo, Norway, (fabianah@math.uio.no).

IDepartment of Mathematics, University of Oslo, P.O. Box 1053, Blindern, N-0316 Oslo, Norway, (proske@math.uio.no).
} 
differential equation of the form

$$
d X(t)=b(t, X(t)) d t+\sigma(t, X(t)) d W_{t}, X_{0}=x \in \mathbb{R}^{d}, 0 \leq t \leq T,
$$

where $W_{t}, 0 \leq t \leq T$ is a $d$-dimensional Wiener process and $b, \sigma$ are continuously differentiable coefficients, the authors in [14] were able to represent (1.1) in a derivative-free form, that is by

$$
E[\Phi(S(T)) \pi]
$$

where $\pi$ is the so called Malliavin weight. Such a representation is also referred to as Bismut-Elworthy-Li formula (BEL-formula) in the literature. See [7] and [8].

An advantage of this method is that the representation in (1.3) does not involve derivatives of $\Phi$ and that it exhibits numerical tractability via efficient use of MonteCarlo simulation. However, a deficiency of this approach is the requirement that the coefficients of the SDE, which describes the dynamics of the asset prices in (1.2), are continuously differentiable. The latter assumption is rather restrictive and excludes the study of interesting financial models. Such models could e.g. pertain to a generalization of the Black-Scholes model with "regime-switching" drift, that is

$$
S_{t}^{x}=x \exp \left(Y_{t}\right)
$$

where

$$
d Y_{t}=\left(b_{1} \chi_{\left\{Y_{t}>R\right\}}+b_{2} \chi_{\left\{Y_{t} \leq R\right\}}\right) d t-\frac{1}{2} \sigma^{2} d t+\sigma d W_{t}
$$

for constants $b_{1}, b_{2}$ and a "threshold" $R$.

Another possible application is to interest rate or commodity markets with a model whose dynamics is given by a generalized Ornstein-Uhlenbeck process with regime switching mean reversion rate, that is

$$
d Y_{t}=\left(a_{1} \chi_{\left\{Y_{t}>R\right\}}+a_{2} \chi_{\left\{Y_{t} \leq R\right\}}\right)\left(b-Y_{t}\right) d t+\sigma d W_{t}
$$

for mean reversion coefficients $a_{1}, a_{2}>0$, a threshold $R$, the long-run average level $b \in \mathbb{R}$, interest rate volatility $\sigma>0$.

In the above models (1.4) and (1.5) the drift coefficients are chosen to be discontinuous and used to capture regime-switching effects which may arise from regulations, credit rating changes, market crashes or other financial disasters.

We mention that a BEL-representation for Wiener process driven SDE's with merely bounded and measurable drift functions as e.g. the one in (1.5) was first obtained in Menoukeu-Pamen et al. [19, Theorem 4.6, Remark 4.7]. To be more precise, for strong solutions $X_{t}, 0 \leq t \leq T$ to SDE's with additive Wiener noise

$$
d X_{t}^{x}=b\left(t, X_{t}^{x}\right) d t+d W_{t},
$$

where $b \in L^{\infty}\left([0, T] \times \mathbb{R}^{d} ; \mathbb{R}^{d}\right)$, the authors prove, for bounded Borel measurable $\Phi$ and bounded open sets $U \subset \mathbb{R}^{d}$, that

$$
\frac{\partial}{\partial x} E\left[\Phi\left(X_{T}^{x}\right)\right]=E\left[\Phi\left(X_{T}^{x}\right) \pi\right]^{*}
$$

for all $x \in U$ a.e., where the Malliavin weight $\pi$ is

$$
\int_{0}^{T} a(s)\left(\frac{\partial}{\partial x} X_{s}^{x}\right)^{*} d W_{s} .
$$


Here the derivatives appearing on both sides of (1.6) are Sobolev derivatives on $U$, $a:[0, T] \longrightarrow \mathbb{R}$ is a bounded Borel measurable function with $\int_{0}^{T} a(s) d s=1$ and $*$ denotes transposition. See also the related articles [4], [3], [23] and the references therein.

Using techniques from Malliavin calculus and arguments of a "local time variational calculus" as recently developed in the series of works [5], [6], [2] in the case of fractional Brownian motion, we aim at obtaining in this paper an extension of the above mentioned results to the case of fractional Brownian motion driven singular SDE's. More precisely, we want to derive a BEL-formula of the type (1.6) with respect to strong solutions to SDE's of the form

$$
d X_{t}=b\left(t, X_{t}\right) d t+d B_{t}^{H}, X_{0}=x, 0 \leq t \leq T,
$$

where $B_{t}^{H}, 0 \leq t \leq T$ is a $d$-dimensional fractional Brownian motion with Hurst parameter $H \in\left(0, \frac{1}{2}\right)$ and where the vector field $b$ is singular in the sense that

$$
b \in L_{\infty, \infty}^{1, \infty}:=L^{1}\left(\mathbb{R}^{d} ; L^{\infty}\left([0, T] ; \mathbb{R}^{d}\right)\right) \cap L^{\infty}\left(\mathbb{R}^{d} ; L^{\infty}\left([0, T] ; \mathbb{R}^{d}\right)\right) .
$$

As an application of the techniques used in connection with the BEL-formula, we also wish to study a Black-Scholes model with "turbulent" stochastic volatility, where the dynamics of stock prices is described by the (singular) SDE

$$
d X_{t}^{x}=\mu X_{t}^{x} d t+\sigma_{t} X_{t}^{x} d W_{t}, X_{0}^{x}=x, 0 \leq t \leq T .
$$

Here $W_{t}, 0 \leq t \leq T$ is a one-dimensional Wiener process, $\mu$ the mean return and $\sigma_{t}$ the volatility at time $t$, modeled by means of the SDE

$$
d Y_{t}^{y}=b\left(t, Y_{t}^{y}\right) d t+B_{t}^{H}, Y_{0}^{y}=y, 0 \leq t \leq T
$$

for small Hurst parameters $H \in(0,1 / 2)$ and singular vector fields $b \in L_{\infty, \infty}^{1, \infty}$, which can be used as explained above for the modeling of regime switching effects in stock markets. Let us also mention that the choice of fractional Brownian motion with small Hurst parameters $H$ in the latter model, which becomes "rougher" the lower $H$ is, is in fact supported by empirical evidence (see [16]) and useful for the description of stock price volatilities $\sigma_{t}$ in "turbulent" stock markets.

Finally, we also point out the interesting work [13], where the authors derived BEL-formulas for (functional) SDE's driven by fractional Brownian motion with Hurst parameters $H \in(0,1)$ in the case of differentiable vector fields, which they applied to e.g. the study of Harnack type of inequalities.

The paper is organized as follows: In Section 2 we prove a BEL-formula with respect to the SDE (1.7) for $H<\frac{1}{2(d+2)}$. See Theorem 2.2. We then show, in Proposition 2.2, that the BEL-representation has a continuous version, if $H<\frac{1}{2(d+3)}$. Finally, in Section 3 we discuss an application of our techniques used in Section 2 to the sensitivity analysis of prices of options based on a Black-Scholes model with "rough" stochastic volatility (Theorem 3.1). 


\section{Bismut-Elworthy-Li formula}

In this section we aim at deriving a new Bismut-Elworthy-Li type formula with respect to SDE's driven by discontinuous vector fields and a fractional Brownian motion with a Hurst parameter $H<\frac{1}{2}$. We also propose a stock price model with "rough" stochastic volatility, which allows for the description of regime switching effects with respect to volatility data caused e.g. by economical crises, political changes or other shocks on markets. Here, regime switching effects are modeled by means of singular coefficients of SDE's driven by a fractional Brownian motion. On the other hand, the "roughness" of the volatility paths in the sense of paths with low Hölder regularity is described through the driving fractional noise of such SDE's. Further, we also prove a BEL-representation for the delta of an option with respect to that model.

In what follows, let us consider a fractional Brownian motion $B_{t}^{H}, t \geq 0$ with Hurst parameter $H \in(0,1)$ on some complete probability space $(\Omega, \mathcal{F}, \mu)$, which is (in the 1 -dimensional case) a centered Gaussian process with a covariance structure $R_{H}(t, s)$ of the form

$$
R_{H}(t, s)=E\left[B_{t}^{H} B_{s}^{H}\right]=\frac{1}{2}\left(s^{2 H}+t^{2 H}-|t-s|^{2 H}\right)
$$

for all $t, s \geq 0$. See the Appendix. In the special case, when $H=\frac{1}{2}$ the fractional Brownian motion coincides with a Wiener process.

We also recall that the fractional Brownian motion is self-similar, that is

$$
\left\{B_{\alpha t}^{H}\right\}_{t \geq 0} \stackrel{\operatorname{law}}{=}\left\{\alpha^{H} B_{t}^{H}\right\}_{t \geq 0}
$$

for all $\alpha>0$. Further, $B^{H}$ has a version with paths, which are $(H-\varepsilon)$-Hölder continuous for all $\varepsilon \in(0, H)$. Another property satisfied by $B^{H}$, which actually rather complicates the study of fractional Brownian motion, is that it is neither a Markov process nor a semimartingale, when $H \neq \frac{1}{2}$. See e.g. [20] and the references therein for further information on the fractional Brownian motion.

In this Section, we consider for $H<\frac{1}{2}$ the SDE

$$
d X_{t}^{x}=b\left(t, X_{t}^{x}\right) d t+d B_{t}^{H}, X_{0}^{x}=x, 0 \leq t \leq T .
$$

We mention that $B^{H}$ in this case has the representation

$$
B_{t}^{H}=\int_{0}^{t} K_{H}(t, s) I_{d \times d} d B_{s}
$$

for a $d$-dimensional Brownian motion $B$., where $I_{d \times d} \in \mathbb{R}^{d \times d}$ is the unit matrix and $K_{H}$ the kernel as given in (4.2) in the Appendix.

In the sequel, we also need the following notation for function spaces:

$$
\begin{aligned}
L_{\infty}^{1} & :=L^{1}\left(\mathbb{R}^{d} ; L^{\infty}\left([0, T] ; \mathbb{R}^{d}\right)\right), \\
L_{\infty}^{\infty}: & =L^{\infty}\left(\mathbb{R}^{d} ; L^{\infty}\left([0, T] ; \mathbb{R}^{d}\right)\right), \\
L_{\infty, \infty}^{1, \infty}: & =L_{\infty}^{1} \cap L_{\infty}^{\infty} .
\end{aligned}
$$

We have the following result for the existence and uniqueness of strong solutions to the SDE (2.1) which is due to [5] (compare also the results in [9] and [21], which cannot be used to treat the case $b \in L_{\infty, \infty}^{1, \infty}$ for $\left.d>1\right)$ :

THEOREM 2.1. Let $b \in L_{\infty, \infty}^{1, \infty}$. Then if $H<\frac{1}{2(d+2)}$ there exists a unique (global) strong solution $X^{x}$ of the SDE (2.1). Moreover, for every $x \in \mathbb{R}^{d}, t \in[0, T] X_{t}^{x}$ is Malliavin 
differentiable in the direction of the Brownian motion $B$ in (2.2) and $X_{t}$ is locally Sobolev differentiable $\mu$-a.e.

That is, more precisely,

$$
X_{t} \in \bigcap_{p \geq 2} L^{2}\left(\Omega ; W^{1, p}(U)\right)
$$

for bounded and open sets $U \subset \mathbb{R}^{d}$. results:

In preparation of our main result (Theorem 2.2), we also need a series of auxiliary Lemma 2.1. Let $b \in C_{c}^{\infty}\left((0, T) \times \mathbb{R}^{d}\right)$. Fix integers $p \geq 2$. Then, if $H<\frac{1}{2(d+2)}$, we have

$$
\sup _{x \in \mathbb{R}^{d}} E\left[\left\|\frac{\partial}{\partial x} X_{t}^{x}\right\|^{p}\right] \leq C_{p, H, d, T}\left(\|b\|_{L_{\infty}^{\infty}},\|b\|_{L_{\infty}^{1}}\right)<\infty
$$

for some continuous function $C_{p, H, d, T}:[0, \infty)^{2} \longrightarrow[0, \infty)$.

Proof. See [5].

Lemma 2.2. Let $H<\frac{1}{2(d+2)}, b \in L_{\infty, \infty}^{1, \infty}$. Further, let $X^{n}, n \geq 1$ be the sequence of strong solutions to (2.1) associated with functions $b_{n} \in C_{c}^{\infty}\left((0, T) \times \mathbb{R}^{d}\right), n \geq 1$ such that

$$
\begin{aligned}
b_{n}(t, x) & \underset{n \longrightarrow \infty}{\longrightarrow} b(t, x)(t, x)-\text { a.e. }, \\
& \sup _{n \geq 1}\left\|b_{n}\right\|_{L_{\infty}^{1}}<\infty
\end{aligned}
$$

and

$$
|b(t, x)| \leq M<\infty, n \geq 1 \text { a.e. for some constant } M .
$$

Fix $t \in[0, T]$ and $x \in \mathbb{R}^{d}$. Then there exists a $\beta \in(0,1 / 2)$ such that

$$
\sup _{n \geq 1} \int_{0}^{t} \int_{0}^{t} \frac{E\left[\left\|D_{\theta} X_{t}^{n}-D_{\theta^{\prime}} X_{t}^{n}\right\|^{2}\right]}{\left|\theta-\theta^{\prime}\right|^{1+2 \beta}} d \theta^{\prime} d \theta \leq \sup _{n \geq 1} C_{H, d, T}\left(\left\|b_{n}\right\|_{L_{\infty}^{\infty}},\left\|b_{n}\right\|_{L_{\infty}^{1}}\right)<\infty
$$

and

$$
\sup _{n \geq 1}\left\|D \cdot X_{t}^{n}\right\|_{L^{2}(\Omega \times[0, T])} \leq \sup _{n \geq 1} C_{H, d, T}\left(\left\|b_{n}\right\|_{L_{\infty}^{\infty}},\left\|b_{n}\right\|_{L_{\infty}^{1}}\right)<\infty
$$

for some continuous function $C_{H, d, T}:[0, \infty)^{2} \longrightarrow[0, \infty)$.

Proof. See [5].

Proposition 2.1. Let $X^{x, n}, n \geq 1$ be a sequence of strong solutions as in Lemma 2.2 and $X$. the strong solution to (2.1). Then

$$
X_{t}^{x, n} \underset{n \longrightarrow \infty}{\longrightarrow} X_{t}^{x} \text { in } L^{2}(\Omega)
$$


for all $t, x$.

Proof. See [5].

Lemma 2.3. Let $U \subset \mathbb{R}^{d}$ be an open and bounded subset. Consider the sequence $X^{x, n}, n \geq 1$ in Proposition 2.1. Then

$$
\frac{\partial}{\partial x} X^{\cdot, n} \underset{n \longrightarrow \infty}{\longrightarrow} \frac{\partial}{\partial x} X
$$

in $L^{2}([0, T] \times \Omega \times U)$ weakly.

Proof. This result is a consequence of Proposition 2.1 and the estimate in Lemma 2.1.

We are coming now to the main result of our article:

Theorem 2.2 (Bismut-Elworthy-Li formula). Let $H<\frac{1}{2(d+2)}$ and let $X^{x}$. be the unique strong solution to the SDE

$$
d X_{t}^{x}=b\left(t, X_{t}^{x}\right) d t+d B_{t}^{H}, X_{0}^{x}=x, 0 \leq t \leq T
$$

for $b \in L_{\infty, \infty}^{1, \infty}$. Further, assume that $U$ is a bounded and open subset of $\mathbb{R}^{d}$ and $\Phi: \mathbb{R}^{d} \longrightarrow$ $\mathbb{R}$ a Borel measurable function such that

$$
\Phi\left(X_{T}^{\cdot}\right) \in L^{2}(\Omega \times U, \mu \times d x) .
$$

. In addition, consider a bounded Borel measurable function $a:[0, T] \longrightarrow \mathbb{R}$ such that

$$
\int_{0}^{T} a(s) d s=1
$$

Then

$$
\frac{\partial}{\partial x} E\left[\Phi\left(X_{T}^{x}\right)\right]=C_{H} E\left[\Phi\left(X_{T}^{x}\right) \int_{0}^{T} u^{-H-\frac{1}{2}} \int_{u}^{T} a(s-u)(s-u)^{\frac{1}{2}-H} s^{H-\frac{1}{2}}\left(\frac{\partial}{\partial x} X_{s-u}^{x}\right)^{*} d B_{s} d u\right]^{*}
$$

for all $x \in U$ a.e., $0<t \leq T$, where $*$ denotes the transposition of matrices and where $C_{H}=1 /\left(c_{H} \Gamma\left(\frac{1}{2}+H\right) \Gamma\left(\frac{1}{2}-H\right)\right)$ for

$$
c_{H}=\left(\frac{2 H}{(1-2 H) B(1-2 H, H+1 / 2)}\right)^{1 / 2} .
$$

Here $\Gamma$ and $B$ are the Gamma and Beta function, respectively.

REMARK 2.1. Let $\mathcal{P}$ be the predictable $\sigma$-algebra with respect to the $\mu$-augmented filtration $\left\{\mathcal{F}_{t}\right\}_{0 \leq t \leq T}$ generated by $B^{H}$. Then $\frac{\partial}{\partial x} X_{t}^{x}, 0 \leq t \leq T$ on the right hand side of Theorem 2.2 stands for a process $Y:[0, T] \times \Omega \times U \longrightarrow \mathbb{R}^{d \times d}$ in $L^{2}([0, T] \times \Omega \times U, \mathcal{P} \otimes$ $\left.\mathcal{B}(U) ; \mathbb{R}^{d \times d}\right)$ such that $Y_{t}^{\cdot}(\omega)$ is the Sobolev derivative of $X_{t}(\omega)(t, \omega)$-a.e.

REMARK 2.2. The condition of boundedness of the Hurst parameter by the function $\left(d \mapsto \frac{1}{2(d+2)}\right)$ poses some serious questions on the applicability of the previous result. The results in [16] show, see also section 3, that when the volatility is modeled as a one dimensional process the restriction can accommodate many of the observed time series. When $d>2$ the previous result as it is is not directly applicable and two potential solutions are as follows: 
- The strength of the previous result lies in its applicability to very singular vector fields. This, not surprisingly, is also the reason for the restriction on $H$ as a function of the dimension, see [5]. Thus a possible solution would be to restrict the class of vector fields that are covered by the previous theorem or by working in Besov spaces, in the spirit of [9], where the interplay between the regularity of the vector field and the roughness of the driving noise is much more explicit. This is in fact a work in progress and an extension to the results in [5].

- Provided one can accommodate a multiplicative volatility of volatility (vol-ofvol), then a Black and Scholes type model where the volatility is driven by rough one-dimensional volatility is a very rich model that can accommodate many of the stylized facts observed in the markets. This is in principle possible to achieve but at the cost of either strong smoothness requirement on the vol-of-vol or the use of the theory of rough paths in order to give a meaning to integrals with respect to $\mathrm{fBm}$ with $H<\frac{1}{2}$. At this moment in time it is not clear to us how to integrate the last solution with the method used in the proof of the theorem.

Proof. Let $\Phi \in C_{c}^{\infty}\left(\mathbb{R}^{d}\right)$ and choose a sequence of functions $b_{n} \in C_{c}^{\infty}\left((0, T) \times \mathbb{R}^{d}\right)$, which approximates the vector field $b$ in the sense of (2.3), (2.4) and (2.5).

Denote by $X^{s, x, n}$ the unique strong solution to

$$
d X_{t}^{s, x, n}=b_{n}\left(t, X_{t}^{s, x, n}\right) d t+d B_{t}^{H}, X_{s}^{s, x, n}=x, s \leq t \leq T
$$

for all $n$. Since $b_{n} \in C_{c}^{\infty}\left((0, T) \times \mathbb{R}^{d}\right)$, it follows that there exists a $\Omega^{*}$ with $\mu\left(\Omega^{*}\right)=1$ such that for all $\omega \in \Omega^{*}, 0 \leq s \leq t \leq T$

$$
\left(x \mapsto X_{t}^{s, x, n}(\omega)\right) \in C^{\infty}\left(\mathbb{R}^{d}\right) .
$$

See e.g. [17].

The latter and dominated convergence then give

$$
\frac{\partial}{\partial x} E\left[\Phi\left(X_{T}^{x, n}\right)\right]=E\left[\Phi^{\prime}\left(X_{T}^{x, n}\right) \frac{\partial}{\partial x} X_{T}^{x, n}\right]
$$

where $\Phi^{\prime}$ is the derivative of $\Phi$ and $X_{t}^{x, n}=X_{t}^{0, x, n}$. On the other hand, we have that for all $0 \leq s \leq t \leq T, x \in U$

$$
X_{t}^{x, n}=X_{t}^{s, X_{s}^{x, n}, n} \text { a.e. }
$$

So we obtain that

$$
\frac{\partial}{\partial x} E\left[\Phi\left(X_{T}^{x, n}\right)\right]=E\left[\Phi^{\prime}\left(X_{T}^{x, n}\right) \frac{\partial}{\partial x} X_{T}^{s, X_{s}^{x, n}, n} \frac{\partial}{\partial x} X_{s}^{x, n}\right] .
$$

We also know that the Malliavin derivative $D^{H} X_{t}^{s, x, n}$ of $X_{t}^{s, x, n}$ in the direction of $B^{H}$ exists and satisfies the equation

$$
D_{u}^{H} X_{t}^{s, x, n}=\int_{u}^{t} b_{n}^{1}\left(t, X_{r}^{s, x, n}\right) D_{u}^{H} X_{r}^{s, x, n} d r+\chi_{(s, t]}(u) I_{d \times d},
$$

where $I_{d \times d}$ is the identity matrix. Further, we see that $\frac{\partial}{\partial x} X^{u, X_{u}^{x, n}, n}$ solves the same equation for $s=0$. Therefore, we obtain by uniqueness of solutions that

$$
D_{u}^{H} X_{t}^{x, n}=\frac{\partial}{\partial x} X_{t}^{u, X_{u}^{x, n}, n} \quad \text { a.e. }
$$


Hence

$$
\frac{\partial}{\partial x} E\left[\Phi\left(X_{T}^{x, n}\right)\right]=E\left[\Phi^{\prime}\left(X_{T}^{x, n}\right) D_{s}^{H} X_{T}^{x, n} \frac{\partial}{\partial x} X_{s}^{x, n}\right]
$$

Let $\varphi \in C_{c}^{\infty}(U)$. Then

$$
-\int_{U} E\left[\Phi\left(X_{T}^{x, n}\right)\right] \frac{\partial}{\partial x} \varphi(x) d x=\int_{U} \varphi(x) E\left[\Phi^{\prime}\left(X_{T}^{x, n}\right) D_{s}^{H} X_{T}^{x, n} \frac{\partial}{\partial x} X_{s}^{x, n}\right] d x .
$$

Further, using the fact that the function $a$ sums up to one combined with the chain rule for $D^{H}$ (see [20]), we obtain that

$$
\begin{aligned}
& -\int_{U} E\left[\Phi\left(X_{T}^{x, n}\right)\right] \frac{\partial}{\partial x} \varphi(x) d x \\
= & \int_{U} \varphi(x) E\left[\int_{0}^{T}\left\{a(s) \Phi^{\prime}\left(X_{T}^{x, n}\right) D_{s}^{H} X_{T}^{x, n} \frac{\partial}{\partial x} X_{s}^{x, n}\right\} d s\right] d x \\
= & \int_{U} \varphi(x) E\left[\int_{0}^{T}\left\{a(s) D_{s}^{H} \Phi\left(X_{T}^{x, n}\right) \frac{\partial}{\partial x} X_{s}^{x, n}\right\} d s\right] d x
\end{aligned}
$$

On the other hand, Proposition 5.2.1 and p. 285 in [20] shows that

$$
D_{s}^{H} \Phi\left(X_{T}^{x, n}\right)=C s^{\frac{1}{2}-H}\left(\int_{s}^{T}(u-s)^{-H-\frac{1}{2}} u^{H-\frac{1}{2}} D_{u} \Phi\left(X_{T}^{x, n}\right) d u\right) .
$$

for a constant $C$ depending on $H$. D. stands here for the Malliavin derivative in the direction of the Brownian motion $B$.

Hence, we obtain by substitution (first for $u$ substituted by $u+s$ in the above relation and then for $s$ by $s-u$ in the next step), Fubini's theorem and the duality formula with respect to the Malliavin derivative $D$. that

$$
\begin{aligned}
& -\int_{U} E\left[\Phi\left(X_{T}^{x, n}\right)\right] \frac{\partial}{\partial x} \varphi(x) d x \\
= & C \int_{U} \varphi(x) E\left[\int _ { 0 } ^ { T } \left\{a(s) C s^{\frac{1}{2}-H}\right.\right. \\
& \left.\left.\times\left(\int_{s}^{T}(u-s)^{-H-\frac{1}{2}} u^{H-\frac{1}{2}} D_{u} \Phi\left(X_{T}^{x, n}\right) d u\right) \frac{\partial}{\partial x} X_{s}^{x, n}\right\} d s\right] d x \\
= & C \int_{U} \varphi(x) E\left[\int_{0}^{T} u^{-H-\frac{1}{2}}\right. \\
& \left.\times \int_{u}^{T} a(s-u)(s-u)^{\frac{1}{2}-H} s^{H-\frac{1}{2}} D_{s} \Phi\left(X_{T}^{x, n}\right) \frac{\partial}{\partial x} X_{s-u}^{x, n} d s d u\right] d x \\
= & C \int_{U} \varphi(x) E\left[\Phi\left(X_{T}^{x, n}\right)\right. \\
& \left.\times \int_{0}^{T} u^{-H-\frac{1}{2}} \int_{u}^{T} a(s-u)(s-u)^{\frac{1}{2}-H} s^{H-\frac{1}{2}}\left(\frac{\partial}{\partial x} X_{s-u}^{x, n}\right)^{*} d B_{s} d u\right]^{*} d x \\
= & I_{1}(n)+I_{2}(n),
\end{aligned}
$$

where

$$
I_{1}(n):=C \int_{U} \varphi(x) E\left[\left(\Phi\left(X_{T}^{x, n}\right)-\Phi\left(X_{T}^{x}\right)\right)\right.
$$




$$
\left.\times \int_{0}^{T} u^{-H-\frac{1}{2}} \int_{u}^{T} a(s-u)(s-u)^{\frac{1}{2}-H} s^{H-\frac{1}{2}}\left(\frac{\partial}{\partial x} X_{s-u}^{x, n}\right)^{*} d B_{s} d u\right]^{*} d x
$$

and

$$
\begin{aligned}
I_{2}(n): & =C \int_{U} \varphi(x) E\left[\Phi\left(X_{T}^{x}\right) \int_{0}^{T} u^{-H-\frac{1}{2}}\right. \\
& \left.\times \int_{u}^{T} a(s-u)(s-u)^{\frac{1}{2}-H} s^{H-\frac{1}{2}}\left(\frac{\partial}{\partial x} X_{s-u}^{x, n}\right)^{*} d B_{s} d u\right]^{*} d x \\
= & C \int_{U} \varphi(x) E\left[\Phi\left(X_{T}^{x}\right) \int_{0}^{T} u^{-H-\frac{1}{2}}\right. \\
& \left.\times \int_{u}^{T} a(s-u)(s-u)^{\frac{1}{2}-H} s^{H-\frac{1}{2}}\left(\frac{\partial}{\partial x} X_{s-u}^{x}\right)^{*} d B_{s} d u\right]^{*} d x \\
& +I_{3}(n),
\end{aligned}
$$

where

$$
\begin{aligned}
& I_{3}(n) \\
: & =C \int_{U} \varphi(x) E\left[\Phi\left(X_{T}^{x}\right) \int_{0}^{T} u^{-H-\frac{1}{2}} \int_{u}^{T} a(s-u)(s-u)^{\frac{1}{2}-H} s^{H-\frac{1}{2}}\right. \\
& \left.\times\left\{\left(\frac{\partial}{\partial x} X_{s-u}^{x, n}\right)^{*}-\left(\frac{\partial}{\partial x} X_{s-u}^{x}\right)^{*}\right\} d B_{s} d u\right]^{*} d x .
\end{aligned}
$$

It follows from Fubini's theorem, Hölder's inequality, the Itô isometry, Lemma 2.1, Lemma 2.1 and dominated convergence that

$$
\begin{aligned}
& \left\|I_{1}(n)\right\| \\
\leq & C\|\varphi\|_{\infty} \int_{U}\left(E\left[\left|\Phi\left(X_{T}^{x, n}\right)-\Phi\left(X_{T}^{x}\right)\right|^{2}\right]\right)^{1 / 2} \\
& \times\left(\int_{0}^{T} s^{2 H-1} E\left[\left(\int_{0}^{s} u^{-H-\frac{1}{2}}|a(s-u)|(s-u)^{\frac{1}{2}-H}\left\|\frac{\partial}{\partial x} X_{s-u}^{x, n}\right\| d u\right)^{2}\right] d s\right)^{1 / 2} d x \\
\leq & C\|\varphi\|_{\infty} \int_{U}\left(E\left[\left|\Phi\left(X_{T}^{x, n}\right)-\Phi\left(X_{T}^{x}\right)\right|^{2}\right]\right)^{1 / 2}\left(\int_{0}^{T} s^{2 H-1}\right. \\
& \times \int_{0}^{s} \int_{0}^{s} u_{1}^{-H-\frac{1}{2}}\left|a\left(s-u_{1}\right)\right|\left(s-u_{1}\right)^{\frac{1}{2}-H} u_{2}^{-H-\frac{1}{2}}\left|a\left(s-u_{2}\right)\right|\left(s-u_{2}\right)^{\frac{1}{2}-H} \\
& \left.\left.\times E\left[\left\|\frac{\partial}{\partial x} X_{s-u_{1}}^{x, n}\right\|\right]^{2}\right]^{1 / 2} E\left[\left\|\frac{\partial}{\partial x} X_{s-u_{2}}^{x, n}\right\|^{2}\right]^{1 / 2} d u_{1} d u_{2} d s\right)^{1 / 2} d x \\
= & C\|\varphi\|_{\infty}\left(E\left[\left|\Phi\left(X_{T}^{x, n}\right)-\Phi\left(X_{T}^{x}\right)\right|^{2}\right]\right)^{1 / 2}\left(\int_{0}^{T} s^{2 H-1}\right. \\
& \left.\times\left(\int_{0}^{s} u^{-H-\frac{1}{2}}|a(s-u)|(s-u)^{\frac{1}{2}-H} E\left[\left\|\frac{\partial}{\partial x} X_{s-u}^{x, n}\right\|^{2}\right]^{1 / 2} d u\right)^{2} d s\right)^{1 / 2} d x \\
\leq & C\|\varphi\|_{\infty} \int_{U}\left(E\left[\left|\Phi\left(X_{T}^{x, n}\right)-\Phi\left(X_{T}^{x}\right)\right|^{2}\right]\right)^{1 / 2} d x\left(\int_{0}^{T} s^{2 H-1}\right. \\
& \left.\times \sup _{n \geq 1} C_{1,2 H, d, T}\left(\left\|b_{n}\right\|_{L_{\infty}^{\infty}},\left\|b_{n}\right\|_{L_{\infty}^{1}}\right)^{1 / 4}\left(\int_{0}^{s} u^{-H-\frac{1}{2}}|a(s-u)|(s-u)^{\frac{1}{2}-H} d u\right)^{2} d s\right)^{1 / 2}
\end{aligned}
$$




$$
\begin{aligned}
\leq & C\|\varphi\|_{\infty} \int_{U}\left(E\left[\left|\Phi\left(X_{T}^{x, n}\right)-\Phi\left(X_{T}^{x}\right)\right|^{2}\right]\right)^{1 / 2} d x\left(\int_{0}^{T} s^{H-\frac{1}{2}} d s\right)^{1 / 2} \\
& \longrightarrow 0,
\end{aligned}
$$

where used the boundedness of the function $a$ in the last estimate.

By applying the Clark-Ocone formula (see e.g. [20]) in combination with Itô's isometry and the chain rule for the Malliavin derivative, we see that

$$
\begin{aligned}
& I_{3}(n) \\
: & =C \int_{U} \varphi(x) E\left[E\left[\Phi\left(X_{T}^{x}\right)\right] \int_{0}^{T} u^{-H-\frac{1}{2}} \int_{u}^{T} a(s-u)(s-u)^{\frac{1}{2}-H} s^{H-\frac{1}{2}}\right. \\
& \left.\times\left\{\left(\frac{\partial}{\partial x} X_{s-u}^{x, n}\right)^{*}-\left(\frac{\partial}{\partial x} X_{s-u}^{x}\right)^{*}\right\} d B_{s} d u\right]^{*} d x \\
& +C \int_{U} \varphi(x) E\left[\int_{0}^{T} u^{-H-\frac{1}{2}} \int_{u}^{T} a(s-u)(s-u)^{\frac{1}{2}-H} s^{H-\frac{1}{2}} D_{s} \Phi\left(X_{T}^{x}\right)\right. \\
& \left.\times\left\{\frac{\partial}{\partial x} X_{s-u}^{x, n}-\frac{\partial}{\partial x} X_{s-u}^{x}\right\}^{*} d s d u\right]^{*} d x \\
= & C \int_{U} \varphi(x) E\left[\int_{0}^{T} u^{-H-\frac{1}{2}} \int_{u}^{T} a(s-u)(s-u)^{\frac{1}{2}-H} s^{H-\frac{1}{2}} \Phi^{\prime}\left(X_{T}^{x}\right) D_{s} X_{T}^{x}\right. \\
& \left.\times\left\{\frac{\partial}{\partial x} X_{s-u}^{x, n}-\frac{\partial}{\partial x} X_{s-u}^{x}\right\}^{*} d s d u\right]^{*} d x .
\end{aligned}
$$

Then using Lemma 2.3, Lemma 2.2 and dominated convergence in connection with Lemma 2.1, we find that

$$
\left\|I_{3}(n)\right\|_{n \longrightarrow \infty}^{\longrightarrow} 0 .
$$

Here we mention that $D \cdot X_{T}^{*}$ used above stands for a weak limit of a subsequence of $D . X_{T}^{\cdot, n}, n \geq 1$ in $L^{2}([0, T] \times \Omega \times U)$ such that $D . X_{T}^{x}$ is a representative of the Malliavin derivative of $X_{T}^{x}$ for almost all $x$ in $U$. The latter however is a consequence of Lemma 1.2.3 in [20] in connection with Lemma 2.1, dominated convergence and the bound (2.6), which is independent of $x$.

Similarly, we also obtain that

$$
-\int_{U} E\left[\Phi\left(X_{T}^{x, n}\right)\right] \frac{\partial}{\partial x} \varphi(x) d x \underset{n \longrightarrow \infty}{\longrightarrow} \int_{U} E\left[\Phi\left(X_{T}^{x}\right)\right] \frac{\partial}{\partial x} \varphi(x) d x .
$$

So

$$
\begin{aligned}
& -\int_{U} E\left[\Phi\left(X_{T}^{x}\right)\right] \frac{\partial}{\partial x} \varphi(x) d x \\
= & C \int_{U} \varphi(x) E\left[\Phi\left(X_{T}^{x}\right)\right. \\
& \left.\times \int_{0}^{T} u^{-H-\frac{1}{2}} \int_{u}^{T} a(s-u)(s-u)^{\frac{1}{2}-H} s^{H-\frac{1}{2}}\left(\frac{\partial}{\partial x} X_{s-u}^{x}\right)^{*} d B_{s} d s\right]^{*} d x
\end{aligned}
$$

Finally, we can apply the monotone class theorem in connection with dominated convergence and the Cauchy-Schwarz inequality and verify the latter relation for Borel measurable functions $\Phi: \mathbb{R}^{d} \longrightarrow \mathbb{R}$ such that

$$
\Phi\left(X_{T}^{*}\right) \in L^{2}(\Omega \times U, \mu \times d x) .
$$


Hence the result follows.

In financial applications the right hand side of relation (2.7), say $M$ may be interpreted as a sensitivity measure- known as delta- for changes of the fair value of an option with payoff function $\Phi$ and underlying $d$ stock price processes $X^{x}$. (under a change of measure) with respect to the initial prices $x \in \mathbb{R}^{d}$ of the stocks. The quantity $M$, is a priori for $H<\frac{1}{2(d+2)}$ only defined for almost all initial values $x$. In practice, however, where a trader is interested in sensitivities with respect to specific initial prices of the stocks, the choice of $M$ as a sensitivity measure would be not satisfactory. On the other hand, in order to make sense of $M$ as a delta for all $x \in \mathbb{R}^{d}$, one can in fact choose a version of $M$, which is continuous and hence defined for all $x$. It turns out, however that such a version of $M$ exists, if the Hurst parameter is allowed to be a little bit smaller than in Theorem 2.2, that is $H<\frac{1}{2(d+3)}$. See Proposition 2.2, whose proof requires the following new estimate, which is based on Theorem 4.2:

Lemma 2.4. Let $b \in C_{c}^{\infty}\left((0, T) \times \mathbb{R}^{d}\right)$. Fix integers $p \geq 2$. Then, if $H<\frac{1}{2(d+3)}$, we have

$$
\sup _{x \in \mathbb{R}^{d}} E\left[\left\|\frac{\partial^{2}}{\partial x^{2}} X_{t}^{s, x}\right\|^{p}\right] \leq C_{p, H, d, T}\left(\|b\|_{L_{\infty}^{\infty}},\|b\|_{L_{\infty}^{1}}\right)<\infty
$$

for some continuous function $C_{p, H, d, T}:[0, \infty)^{2} \longrightarrow[0, \infty)$.

Proof. Since the stochastic flow associated with the smooth vector field $b$ is smooth, too (compare to e.g. [17]), we obtain that

$$
\frac{\partial}{\partial x} X_{t}^{s, x}=I_{d \times d}+\int_{s}^{t} \mathrm{D} b\left(u, X_{u}^{s, x}\right) \frac{\partial}{\partial x} X_{u}^{s, x} d u,
$$

where $\mathrm{D} b: \mathbb{R}^{d} \longrightarrow L\left(\mathbb{R}^{d}, \mathbb{R}^{d}\right)$ is the derivative of $b$ with respect to the space variable.

Using Picard iteration, we see that

$$
\frac{\partial}{\partial x} X_{t}^{s, x}=I_{d \times d}+\sum_{m \geq 1} \int_{\Delta_{s, t}^{m}} \mathrm{D} b\left(u_{1}, X_{u_{1}}^{s, x}\right) \ldots \mathrm{D} b\left(u_{m}, X_{u_{m}}^{s, x}\right) d u_{m} \ldots d u_{1},
$$

where

$$
\Delta_{s, t}^{m}=\left\{\left(u_{m}, \ldots u_{1}\right) \in[0, T]^{m}: \theta<u_{m}<\ldots<u_{1}<t\right\} .
$$

By using dominated convergence, we can differentiate both sides with respect to $x$ and get that

$$
\frac{\partial^{2}}{\partial x^{2}} X_{t}^{s, x}=\sum_{m \geq 1} \int_{\Delta_{s, t}^{m}} \frac{\partial}{\partial x}\left[\mathrm{D} b\left(u_{1}, X_{u_{1}}^{s, x}\right) \ldots \mathrm{D} b\left(u_{m}, X_{u_{m}}^{s, x}\right)\right] d u_{m} \ldots d u_{1} .
$$

Then application of the Leibniz and chain rule yields

$$
\begin{aligned}
& \frac{\partial}{\partial x}\left[\mathrm{D} b\left(u_{1}, X_{u_{1}}^{s, x}\right) \ldots \mathrm{D} b\left(u_{m}, X_{u_{m}}^{s, x}\right)\right] \\
= & \sum_{r=1}^{m} \mathrm{D} b\left(u_{1}, X_{u_{1}}^{s, x}\right) \ldots \mathrm{D}^{2} b\left(u_{r}, X_{u_{r}}^{s, x}\right) \frac{\partial}{\partial x} X_{u_{r}}^{s, x} \ldots \mathrm{D} b\left(u_{m}, X_{u_{m}}^{s, x}\right),
\end{aligned}
$$

where $\mathrm{D}^{2} b=\mathrm{D}(\mathrm{D} b): \mathbb{R}^{d} \longrightarrow L\left(\mathbb{R}^{d}, L\left(\mathbb{R}^{d}, \mathbb{R}^{d}\right)\right)$. 
So it follows from (2.9) that

$$
\begin{aligned}
\frac{\partial^{2}}{\partial x^{2}} X_{t}^{s, x}= & \sum_{m_{1} \geq 1} \int_{\Delta_{s, t}^{m_{1}}} \sum_{r=1}^{m_{1}} \mathrm{D} b\left(u_{1}, X_{u_{1}}^{s, x}\right) \ldots \mathrm{D}^{2} b\left(u_{r}, X_{u_{r}}^{s, x}\right) \\
& \times\left(I_{d \times d}+\sum_{m_{2} \geq 1} \int_{\Delta_{s, u_{r}}^{m_{2}}} \mathrm{D} b\left(v_{1}, X_{v_{1}}^{s, x}\right) \ldots \mathrm{D} b\left(v_{m_{2}}, X_{v_{m_{2}}}^{s, x}\right) d v_{m_{2}} \ldots d v_{1}\right) \\
& \times \mathrm{D} b\left(u_{r+1}, X_{u_{r+1}}^{s, x}\right) \ldots \mathrm{D} b\left(u_{m_{1}}, X_{u_{m_{1}}}^{s, x}\right) d u_{m_{1} \ldots d u_{1}} \\
= & \sum_{m_{1} \geq 1} \sum_{r=1}^{m_{1}} \int_{\Delta_{s, t}^{m_{1}}} \mathrm{D} b\left(u_{1}, X_{u_{1}}^{s, x}\right) \ldots \mathrm{D}^{2} b\left(u_{r}, X_{u_{r}}^{s, x}\right) \ldots \mathrm{D} b\left(u_{m_{1}}, X_{u_{m_{1}}}^{s, x}\right) d u_{m_{1}} \ldots d u_{1} \\
& +\sum_{m_{1} \geq 1} \sum_{r=1}^{m_{1}} \sum_{m_{2} \geq 1} \int_{\Delta_{s, t}^{m_{1}}} \int_{\Delta_{s, u_{r}}^{m_{2}}} \mathrm{D} b\left(u_{1}, X_{u_{1}}^{s, x}\right) \ldots \mathrm{D}^{2} b\left(u_{r}, X_{u_{r}}^{s, x}\right) \\
& \times \mathrm{D} b\left(v_{1}, X_{v_{1}}^{s, x}\right) \ldots \mathrm{D} b\left(v_{m_{2}}, X_{v_{m_{2}}}^{s, x}\right) \mathrm{D} b\left(u_{r+1}, X_{u_{r+1}}^{s, x}\right) \ldots \mathrm{D} b\left(u_{m_{1}}, X_{u_{m_{1}}}^{s, x}\right) \\
& d v_{m_{2}} \ldots d v_{1} d u_{m_{1}} \ldots d u_{1} \\
= & : I_{1}+I_{2} .
\end{aligned}
$$

We now aim at applying Lemma 4.3 to the term $I_{2}$ in (2.10) and find that

$$
I_{2}=\sum_{m_{1} \geq 1} \sum_{r=1}^{m_{1}} \sum_{m_{2} \geq 1} \int_{\Delta_{s, t}^{m_{1}+m_{2}}} \mathcal{H}_{m_{1}+m_{2}}^{X}(u) d u_{m_{1}+m_{2}} \ldots d u_{1}
$$

for $u=\left(u_{1}, \ldots, u_{m_{1}+m_{2}}\right)$, where the integrand $\mathcal{H}_{m_{1}+m_{2}}^{X}(u) \in \mathbb{R}^{d} \otimes \mathbb{R}^{d} \otimes \mathbb{R}^{d}$ has entries given by sums of at most $C(d)^{m_{1}+m_{2}}$ summands, each of which is a product of length $m_{1}+m_{2}$ of functions belonging to the class

$$
\left\{\frac{\partial^{j}}{\partial x_{l_{1}} \partial x_{l_{j}}} b^{(i)}\left(u, X_{u}^{s, x}\right), j=1,2, l_{1}, l_{2}, i=1, \ldots, d\right\} .
$$

Here it is crucial to mention that the number of times second order derivatives appear in those products of functions in (2.11) is exactly one. Thus the absolute value of the multi-index $\alpha$ with respect to the total order of derivatives of those products of functions in connection with Proposition 4.4 in the Appendix is given by

$$
|\alpha|=m_{1}+m_{2}+1 .
$$

We now choose $p, c, r \in[1, \infty)$ such that $c p=2^{q}$ for some integer $q$ and $\frac{1}{r}+\frac{1}{c}=1$. Then we can employ Hölder's inequality and Girsanov's theorem (Theorem 4.1) in combination with Lemma 4.2 in the Appendix and get that

$$
\begin{aligned}
& E\left[\left\|I_{2}\right\|^{p}\right] \\
\leq & C\left(\|b\|_{L_{\infty}^{\infty}}\right)\left(\sum_{m_{1} \geq 1} \sum_{r=1}^{m_{1}} \sum_{m_{2} \geq 1} \sum_{i \in I}\left\|\int_{\Delta_{s, t}^{m_{1}+m_{2}}} \mathcal{H}_{i}^{B^{H}}(u) d u_{m_{1}+m_{2}} \ldots d u_{1}\right\|_{L^{2^{q}}(\Omega ; \mathbb{R})}\right)^{p}(2.1
\end{aligned}
$$

where $C:[0, \infty) \longrightarrow[0, \infty)$ is a continuous function. Here $\# I \leq K^{m_{1}+m_{2}}$ for a constant $K=K(d)$ and the integrands $\mathcal{H}_{i}^{B^{H}}(u)$ are of the form

$$
\mathcal{H}_{i}^{B^{H}}(u)=\prod_{l=1}^{m_{1}+m_{2}} h_{l}\left(u_{l}\right), h_{l} \in \Lambda, l=1, \ldots, m_{1}+m_{2}
$$


where

$$
\Lambda:=\left\{\frac{\partial^{j}}{\partial x_{l_{1}} \partial x_{l_{j}}} b^{(i)}\left(u, x+B_{u}^{H}\right), j=1,2, l_{1}, l_{2}, i=1, \ldots, d\right\} .
$$

Also in this case functions with second order derivatives only appear once in those products.

Define

$$
J=\left(\int_{\Delta_{s, t}^{m_{1}+m_{2}}} \mathcal{H}_{i}^{B^{H}}(u) d u_{m_{1}+m_{2}} \ldots d u_{1}\right)^{2^{q}} .
$$

Using again Lemma 4.3 in the Appendix, successively, we obtain that $J$ can be written as a sum of, at most of length $K(q)^{m_{1}+m_{2}}$ with summands of the form

$$
\int_{\Delta_{s, t}^{2^{q}\left(m_{1}+m_{2}\right)}} \prod_{l=1}^{2^{q}\left(m_{1}+m_{2}\right)} f_{l}\left(u_{l}\right) d u_{2^{q}\left(m_{1}+m_{2}\right)} \ldots d u_{1}
$$

where $f_{l} \in \Lambda$ for all $l$.

Here the number of factors $f_{l}$ in the above product, which have a second order derivative, is exactly $2^{q}$. Thus the total order of the derivatives involved in (2.14) in connection with Proposition 4.4 is given by

$$
|\alpha|=2^{q}\left(m_{1}+m_{2}+1\right) .
$$

We can now invoke Theorem 4.2 for $m=2^{q}\left(m_{1}+m_{2}\right)$ and $\varepsilon_{j}=0$ and find that

$$
\begin{aligned}
& \left|E\left[\int_{\Delta_{s, t}^{2^{q}\left(m_{1}+m_{2}\right)}} \prod_{l=1}^{2^{q}\left(m_{1}+m_{2}\right)} f_{l}\left(u_{l}\right) d u_{2^{q}\left(m_{1}+m_{2}\right)} \ldots d u_{1}\right]\right| \\
\leq & C^{m_{1}+m_{2}}\left(\|b\|_{L^{1}\left(\mathbb{R}^{d} ; L^{\infty}\left([0, T] ; \mathbb{R}^{d}\right)\right)}\right)^{2^{q}\left(m_{1}+m_{2}\right)} \\
& \times \frac{\left(\left(2\left(2^{q}\left(m_{1}+m_{2}+1\right)\right) !\right)^{1 / 4}\right.}{\Gamma\left(-H\left(2 d 2^{q}\left(m_{1}+m_{2}\right)+42^{q}\left(m_{1}+m_{2}+1\right)\right)+22^{q}\left(m_{1}+m_{2}\right)\right)^{1 / 2}}
\end{aligned}
$$

for a constant $C$ depending on $H, T, d$ and $q$.

So the latter combined with (2.13) shows that

$$
\begin{aligned}
& E\left[\left\|I_{2}\right\|^{p}\right] \\
\leq & C\left(\|b\|_{L_{\infty}^{\infty}}\right)\left(\sum _ { m _ { 1 } \geq 1 } \sum _ { m _ { 2 } \geq 1 } K ^ { m _ { 1 } + m _ { 2 } } \left(\left(\|b\|_{L^{1}\left(\mathbb{R}^{d} ; L^{\infty}\left([0, T] ; \mathbb{R}^{d}\right)\right)}\right)^{2^{q}\left(m_{1}+m_{2}\right)}\right.\right. \\
& \left.\left.\times \frac{\left(\left(2\left(2^{q}\left(m_{1}+m_{2}+1\right)\right) !\right)^{1 / 4}\right.}{\Gamma\left(-H\left(2 d 2^{q}\left(m_{1}+m_{2}\right)+42^{q}\left(m_{1}+m_{2}+1\right)\right)+22^{q}\left(m_{1}+m_{2}\right)\right)^{1 / 2}}\right)^{1 / 2^{q}}\right)^{p}
\end{aligned}
$$

for a constant $K$ depending on $H, T, d, p$ and $q$. $\frac{1}{2(d+3)}$.

Since $\frac{1}{2(d+3)} \leq \frac{1}{2\left(d+2 \frac{m_{1}+m_{2}+1}{m_{1}+m_{2}}\right)}$ for $m_{1}, m_{2} \geq 1$, the above sum converges, when $H<$

Further, one establishes in the same way a similar estimate for $E\left[\left\|I_{1}\right\|^{p}\right]$. Altogether, the proof follows. 
Using Lemma 2.4, we can obtain the following result:

TheOrEm 2.3. Let $b \in L_{\infty, \infty}^{1, \infty}, H<\frac{1}{2(d+3)}$ and $U \subset \mathbb{R}^{d}$ a bounded and open set. Then for all $0 \leq t \leq T$ we have that

$$
X_{t} \in \bigcap_{p \geq 2} L^{2}\left(\Omega ; W^{2, p}(U)\right) .
$$

In particular, for all $0 \leq t \leq T$ there exists a $\Omega^{*}$ with $\mu\left(\Omega^{*}\right)=1$ such that for all $\omega \in \Omega^{*}$ $\left(x \longmapsto X_{t}^{x}(\omega)\right)$ has a continuous version on $U$.

Proof. Following the ideas of Proposition 4.2 in [19], we approximate $b$ by a sequence of vector fields $b_{n} \in C_{c}^{\infty}\left((0, T) \times \mathbb{R}^{d}\right), n \geq 1$ in the sense of the conditions (2.3), (2.4), (2.5). Let $X^{x, n}, n \geq 1$ be the sequence of strong solutions to (2.1) associated with those functions. Let $\phi \in C_{c}^{\infty}\left(U ; \mathbb{R}^{d}\right)$ and define for fixed $t \in[0, T]$ the sequence of random variables

$$
\left\langle X_{t}^{\cdot, n}, \phi\right\rangle:=\int_{U}\left\langle X_{t}^{x, n}, \phi\right\rangle_{\mathbb{R}^{d}} d x, n \geq 1
$$

By invoking similar arguments as in the proof of Proposition 4.2 in [19], which relies on a compactness criterion for square integrable functionals of Wiener processes (see [10]), in combination with the estimates of Lemma 5.6 in [6] one proves that there exists a subsequence $n_{j}, j \geq 1$ such that

$$
\left\langle X_{t}^{\cdot, n_{j}}, \phi\right\rangle \underset{j \longrightarrow \infty}{\longrightarrow}\left\langle X_{t}^{\cdot}, \phi\right\rangle
$$

in $L^{2}(\Omega)$ strongly for all $\phi \in C_{c}^{\infty}\left(U ; \mathbb{R}^{d}\right)$, where $X_{s}^{x}, 0 \leq s \leq T$ is the strong solution of Theorem 2.1. Note that we also have from Proposition 2.1 that

$$
X_{t}^{x, n} \underset{n \longrightarrow \infty}{\longrightarrow} X_{t}^{x}
$$

in $L^{2}(\Omega)$ strongly.

Further, one gets from Lemma 2.4 that

$$
\begin{aligned}
& \sup _{n \geq 1}\left\|X_{t}^{\cdot, n}\right\|_{L^{2}\left(\Omega ; W^{2, p}(U)\right)}^{2} \\
\leq & \sum_{i=0}^{2}\left(\int_{\mathcal{U} n \geq 1} \sup _{n \geq 1} E\left[\left\|\mathrm{D}^{i} X_{t}^{x, n}\right\|^{p}\right] d x\right)^{\frac{2}{p}}<\infty
\end{aligned}
$$

for $H<\frac{1}{2(d+3)}$.

On the other hand, we know that $L^{2}\left(\Omega ; W^{2, p}(U)\right)$ is a reflexive space for $p>1$. Hence there exists a subsequence $n_{j}, j \geq 1$ such that

$$
X_{t}^{\cdot, n_{j}} \underset{j \longrightarrow \infty}{\longrightarrow} Y
$$

in $L^{2}\left(\Omega ; W^{2, p}(U)\right)$ weakly. For simplicity, suppose $n_{j}, j \geq 1$ coincides with the subsequence in (2.16). In addition, we obtain for all $A \in \mathcal{F}, \phi \in C_{c}^{\infty}\left(U ; \mathbb{R}^{d}\right), \alpha^{(1)}+\ldots+\alpha^{(d)} \leq 2$ with $\alpha^{(i)} \in \mathbb{N}_{0}, i=1, \ldots, d$ that

$$
E\left[1_{A}\left\langle X_{t}^{\cdot, n_{j}}, \frac{\partial^{\alpha^{(1)}+\ldots+\alpha^{(d)}}}{\partial^{\alpha^{(1)}} x_{1} \ldots \partial^{\alpha^{(d)}} x_{d}} \phi\right\rangle\right]
$$




$$
\begin{aligned}
= & (-1)^{\alpha^{(1)}+\ldots+\alpha^{(d)}} E\left[1_{A}\left\langle\frac{\partial^{\alpha^{(1)}+\ldots+\alpha^{(d)}}}{\partial^{\alpha^{(1)}} x_{1} \ldots \partial^{\alpha^{(d)}} x_{1}} X_{t}^{\cdot, n_{j}}, \phi\right\rangle\right] \\
& j \longrightarrow(-1)^{\alpha^{(1)}+\ldots+\alpha^{(d)}} E\left[1_{A}\left\langle\frac{\partial^{\alpha^{(1)}+\ldots+\alpha^{(d)}}}{\partial^{\alpha^{(1)}} x_{1} \ldots \partial^{\alpha^{(d)}} x_{1}} Y, \phi\right\rangle\right] .
\end{aligned}
$$

On the other hand (2.16) also implies that

$$
\begin{aligned}
& E\left[1_{A}\left\langle X_{t}^{\cdot, n_{j}}, \frac{\partial^{\alpha^{(1)}}+\ldots+\alpha^{(d)}}{\partial^{\alpha^{(1)}} x_{1} \ldots \partial^{\alpha^{(d)}} x_{d}} \phi\right\rangle\right] \\
& \underset{j \longrightarrow \infty}{\longrightarrow} E\left[1_{A}\left\langle X_{t}, \frac{\partial^{\alpha^{(1)}}+\ldots+\alpha^{(d)}}{\partial^{\alpha^{(1)}} x_{1} \ldots \partial^{\alpha^{(d)}} x_{d}} \phi\right\rangle\right] .
\end{aligned}
$$

Hence $X_{t} \in L^{2}\left(\Omega ; W^{2, p}(U)\right)$ for all $p \geq 2$.

Denoting by $M=M(x), x \in U$ the right hand side of relation (2.7), we prove that $M$ possesses a continuous version:

Proposition 2.2. Retain the conditions of Theorem 2.3. Let $p>\max (d, 4)$ and $\Phi$ : $\mathbb{R}^{d} \longrightarrow \mathbb{R}$ be a bounded continuous function. Then $M$ has a continuous version on $U$, which is obtained by replacing in $M$ on the right hand side of (2.7) the derivative of the flow by a predictable version $\left\{Y_{t}, 0 \leq t \leq T\right\} \in L^{2}\left([0, T] \times \Omega, d t \times \mu ; W^{1, p}(U)\right)$ with $Y_{t}(\omega) \in$ $C(U)$ for all $(t, \omega)$.

Proof. As before denote by $\mathcal{P}$ the predictable $\sigma$-algebra on $[0, T] \times \Omega$ with respect to $\left\{\mathcal{F}_{t}\right\}_{0 \leq t \leq T}$. Then, by using almost the same proof of Theorem 2.3 combined with Lemma 2.4, one shows that there exists a $\frac{\partial}{\partial x} X: \in L^{2}\left([0, T] \times \Omega, \mathcal{P}, d t \times \mu ; W^{1, p}(U)\right)$ with $p>\max (d, 4)$ such that $\frac{\partial}{\partial x} X_{t}(\omega)$ is the Sobolev derivative of $X_{t}(\omega)$ on $U(t, \omega)$-a.e. So in particular, we see for $\phi \in L^{\infty}(U ; \mathbb{R})$ that

$$
\int_{U} \frac{\partial}{\partial x} X_{t}^{x} \phi(x) d x, 0 \leq t \leq T
$$

is a predictable process. Now let us choose a continuous version $Y_{t}(\omega)$ of $\frac{\partial}{\partial x} X_{t}(\omega)$ for all $(t, \omega)$ (which exists by a classical Sobolev space theory and our assumptions). Then the process

$$
\int_{U} Y_{t}^{x}(\omega) \phi(x) d x, 0 \leq t \leq T
$$

is predictable, too. Let $\delta_{\epsilon, y} \in L^{\infty}\left(\mathbb{R}^{d}\right), \epsilon>0$ be an approximation of the Dirac delta measure in $y \in U$. Further, let $V$ be an open and bounded set with $\bar{V} \subset U$ and $y \in V$. In addition, consider a continuous function $\varsigma$ on $U$ with compact support in $U$ such that $\varsigma(x)=1$ for all $x \in V$. Then

$$
\int_{U} Y_{t}^{x}(\omega) \varsigma(x) \delta_{\epsilon, y}(x) d x \underset{\epsilon \searrow 0}{\longrightarrow} Y_{t}^{y}(\omega)
$$

for all $(t, \omega)$. So $Y_{t}^{y}, 0 \leq t \leq T$ is a predictable process for all $y \in U$.

Using Itô's isometry we find that

$$
\sup _{x \in U} E\left[\left\|\int_{0}^{T} u^{-H-\frac{1}{2}} \int_{u}^{T} a(s)(s-u)^{\frac{1}{2}-H} s^{H-\frac{1}{2}}\left(Y_{s-u}^{x}\right)^{*} d B_{s} d u\right\|^{2}\right]
$$




$$
\begin{aligned}
& =C \sup _{x \in U} E\left[\int_{0}^{T}\left\|\int_{0}^{T} u^{-H-\frac{1}{2}} \chi_{(u, T)}(s) a(s)(s-u)^{\frac{1}{2}-H} s^{H-\frac{1}{2}}\left(Y_{s-u}^{x}\right)^{*} d u\right\|^{2} d s\right] \\
& \leq C \sup _{x \in U} \int_{0}^{T} E\left[\left(\int_{0}^{T} u^{-H-\frac{1}{2}} \chi_{(u, T)}(s)|a(s)|(s-u)^{\frac{1}{2}-H} s^{H-\frac{1}{2}}\left\|Y_{s-u}^{x}\right\| d u\right)^{2}\right] d s .
\end{aligned}
$$

On the other hand, we see that

$$
\begin{aligned}
& E\left[\left(\int_{0}^{T} u^{-H-\frac{1}{2}} \chi_{(u, T)}(s)|a(s)|(s-u)^{\frac{1}{2}-H} s^{H-\frac{1}{2}}\left\|Y_{s-u}^{x}\right\| d u\right)^{2}\right. \\
= & \int_{0}^{T} \int_{0}^{T} u_{1}^{-H-\frac{1}{2}} \chi_{\left(u_{1}, T\right)}(s)|a(s)|\left(s-u_{1}\right)^{\frac{1}{2}-H} s^{H-\frac{1}{2}} \\
& \times u_{2}^{-H-\frac{1}{2}} \chi_{\left(u_{2}, T\right)}(s)|a(s)|\left(s-u_{2}\right)^{\frac{1}{2}-H} s^{H-\frac{1}{2}} \\
& \times E\left[\left\|Y_{s-u_{1}}^{x}\right\|\left\|Y_{s-u_{2}}^{x}\right\|\right] d u_{1} d u_{2} \\
\leq & \int_{0}^{T} \int_{0}^{T} u_{1}^{-H-\frac{1}{2}} \chi_{\left(u_{1}, T\right)}(s)|a(s)|\left(s-u_{1}\right)^{\frac{1}{2}-H} s^{H-\frac{1}{2}} \\
& \times u_{2}^{-H-\frac{1}{2}} \chi_{\left(u_{2}, T\right)}(s)|a(s)|\left(s-u_{2}\right)^{\frac{1}{2}-H} s^{H-\frac{1}{2}} \\
& \times E\left[\left\|Y_{s-u_{1}}^{x}\right\|^{2}\right]^{1 / 2} E\left[\left\|Y_{s-u_{2}}^{x}\right\|^{2}\right]^{1 / 2} d u_{1} d u_{2} .
\end{aligned}
$$

Let $b_{n}, n \geq 1$ be a sequence of smooth functions, which approximates $b$ in the sense of Theorem 2.3. Denote by $X^{x, n}, n \geq 1$ the corresponding solutions. Then it follows from Lemma 2.1 that for all $B \in \mathcal{B}([0 ; T]), G \in \mathcal{B}(U)$ :

$$
\begin{aligned}
& \int_{B} \int_{G} E\left[\left\langle\frac{\partial}{\partial x} X_{t}^{x, n}, \frac{\partial}{\partial x} X_{t}^{x, m}\right\rangle_{\mathbb{R}^{d \times d}}\right] d x d t \\
\leq & \left|\int_{B} \int_{G} E\left[\left\langle\frac{\partial}{\partial x} X_{t}^{x, n}, \frac{\partial}{\partial x} X_{t}^{x, m}\right\rangle_{\mathbb{R}^{d \times d}}\right] d x d t\right| \\
\leq & \int_{B} \int_{G} E\left[\left\|\frac{\partial}{\partial x} X_{t}^{x, n}\right\|^{2}\right]^{1 / 2} E\left[\left\|\frac{\partial}{\partial x} X_{t}^{x, m}\right\|^{2}\right]^{1 / 2} d x d t \\
\leq & \int_{B} \int_{G}\left(C_{2, H, d, T}\left(\left\|b_{n}\right\|_{L_{\infty}^{\infty}},\left\|b_{n}\right\|_{L_{\infty}^{1}}\right)\right)^{1 / 2}\left(C_{2, H, d, T}\left(\left\|b_{m}\right\|_{L_{\infty}^{\infty}},\left\|b_{m}\right\|_{L_{\infty}^{1}}\right)\right)^{1 / 2} d x d t \\
\leq & \int_{B} \int_{G} K d x d t,
\end{aligned}
$$

where $K<\infty$ is a constant only depending on $H, d, T$ and the "size" of $b$. Hence, by using Lemma 2.1 and weak convergence both in $L^{2}\left([0, T] \times \Omega \times U, d t \times \mu \times d x ; \mathbb{R}^{d \times d}\right)$ and $L^{2}\left([0, T] \times \Omega, \mathcal{P}, d t \times \mu ; W^{1, p}(U)\right)$ for suitable subsequences with respect to $n$ and $m$, successively, we see that $t$-a.e, $x$-a.e.

$$
E\left[\left\|Y_{t}^{x}\right\|^{2}\right] \leq C .
$$

Using Fatou's Lemma combined with the continuity of $\left(x \longmapsto Y_{t}^{x}(\omega)\right)$ for all $(t, \omega)$, we also find that $t$-a.e.

$$
E\left[\left\|Y_{t}^{x}\right\|^{2}\right] \leq C
$$


for all $x \in U$. Similarly, one shows that

$$
E\left[\left\|Y_{t}^{x}\right\|^{4}\right] \leq C
$$

for a constant $C<\infty$ for all $x \in U$. So we obtain from (2.17) that

$$
\begin{aligned}
& \sup _{x \in U} E\left[\left(\int_{0}^{T} u^{-H-\frac{1}{2}} \chi_{(u, T)}(s)|a(s)|(s-u)^{\frac{1}{2}-H} s^{H-\frac{1}{2}}\left\|Y_{s-u}^{x}\right\| d u\right)^{2}\right] \\
\leq & C \int_{0}^{T} \int_{0}^{T} u_{1}^{-H-\frac{1}{2}} \chi_{\left(u_{1}, T\right)}(s)\left(s-u_{1}\right)^{\frac{1}{2}-H} s^{H-\frac{1}{2}} \\
& \times u_{2}^{-H-\frac{1}{2}} \chi_{\left(u_{2}, T\right)}(s)\left(s-u_{2}\right)^{\frac{1}{2}-H} s^{H-\frac{1}{2}} d u_{1} d u_{2} \\
= & C \int_{0}^{T} u^{-H-\frac{1}{2}} \int_{u}^{T}(s-u)^{\frac{1}{2}-H} s^{H-\frac{1}{2}} d s d u<\infty
\end{aligned}
$$

So

$$
\sup _{x \in U} E\left[\left\|\int_{0}^{T} u^{-H-\frac{1}{2}} \int_{u}^{T} a(s)(s-u)^{\frac{1}{2}-H} s^{H-\frac{1}{2}}\left(Y_{s-u}^{x}\right)^{*} d B_{s} d u\right\|^{2}\right]<\infty .
$$

Now let $x_{m} \underset{m \longrightarrow \infty}{\longrightarrow} x \in U$. Then

$$
\begin{aligned}
& \| E\left[\Phi\left(Y_{T}^{x_{m}}\right) \int_{0}^{T} u^{-H-\frac{1}{2}} \int_{u}^{T} a(s)(s-u)^{\frac{1}{2}-H} s^{H-\frac{1}{2}}\left(Y_{s-u}^{x_{m}}\right)^{*} d B_{s} d u\right. \\
& \left.\quad-\Phi\left(Y_{T}^{x}\right) \int_{0}^{T} u^{-H-\frac{1}{2}} \int_{u}^{T} a(s)(s-u)^{\frac{1}{2}-H} s^{H-\frac{1}{2}}\left(Y_{s-u}^{x}\right)^{*} d B_{s} d u\right] \| \\
& \leq\left\|I_{1}\right\|+\left\|I_{2}\right\|,
\end{aligned}
$$

where

$$
:=E\left[\left(\Phi\left(Y_{T}^{x_{m}}\right)-\Phi\left(Y_{T}^{x}\right)\right) \int_{0}^{T} u^{-H-\frac{1}{2}} \int_{u}^{T} a(s)(s-u)^{\frac{1}{2}-H} s^{H-\frac{1}{2}}\left(Y_{s-u}^{x_{m}}\right)^{*} d B_{s} d u\right]
$$

and

$$
:=E\left[\Phi\left(Y_{T}^{x}\right) \int_{0}^{T} u^{-H-\frac{1}{2}} \int_{u}^{T} a(s)(s-u)^{\frac{1}{2}-H} s^{H-\frac{1}{2}}\left(\left(Y_{s-u}^{x_{m}}\right)^{*}-\left(Y_{s-u}^{x}\right)^{*}\right) d B_{s} d u\right] .
$$

It follows from Itô's isometry and (2.20) that

$$
\begin{aligned}
& \left\|I_{1}\right\| \\
\leq & E\left[\left(\Phi\left(Y_{T}^{x_{m}}\right)-\Phi\left(Y_{T}^{x}\right)\right)^{2}\right]^{1 / 2} \\
& \times\left(\sup _{x \in U} E\left[\left(\int_{0}^{T} u^{-H-\frac{1}{2}} \chi_{(u, T)}(s)|a(s)|(s-u)^{\frac{1}{2}-H} s^{H-\frac{1}{2}}\left\|Y_{s-u}^{x}\right\| d u\right)^{2}\right]\right)^{1 / 2} \\
\leq & C E\left[\left(\Phi\left(Y_{T}^{x_{m}}\right)-\Phi\left(Y_{T}^{x}\right)\right)^{2}\right]^{1 / 2} .
\end{aligned}
$$


So because of dominated convergence $I_{1}=I_{1}(m) \underset{m \rightarrow \infty}{\longrightarrow} 0$.

On the other hand

$$
\begin{aligned}
& \left\|I_{2}\right\| \\
\leq & C E\left[\left\|\int_{0}^{T} u^{-H-\frac{1}{2}} \int_{u}^{T} a(s)(s-u)^{\frac{1}{2}-H} s^{H-\frac{1}{2}}\left(\left(Y_{s-u}^{x_{m}}\right)^{*}-\left(Y_{s-u}^{x}\right)^{*}\right) d B_{s} d u\right\|^{2}\right]^{1 / 2} \\
\leq & \sup _{x \in U} E\left[\left(\int_{0}^{T} u^{-H-\frac{1}{2}} \chi_{(u, T)}(s)|a(s)|(s-u)^{\frac{1}{2}-H} s^{H-\frac{1}{2}}\left\|Y_{s-u}^{x_{m}}-Y_{s-u}^{x}\right\| d u\right)^{2}\right] \\
& \int_{0}^{T} \int_{0}^{T} u_{1}^{-H-\frac{1}{2}} \chi_{\left(u_{1}, T\right)}(s)|a(s)|\left(s-u_{1}\right)^{\frac{1}{2}-H} s^{H-\frac{1}{2}} \\
& \times u_{2}^{-H-\frac{1}{2}} \chi_{\left(u_{2}, T\right)}(s)|a(s)|\left(s-u_{2}\right)^{\frac{1}{2}-H} s^{H-\frac{1}{2}} \\
& \times E\left[\left\|Y_{s-u_{1}}^{x}-Y_{s-u_{1}}^{x_{m}}\right\|^{2}\right]^{1 / 2} E\left[\left\|Y_{s-u_{2}}^{x}-Y_{s-u_{2}}^{x_{m}}\right\|^{2}\right]^{1 / 2} d u_{1} d u_{2} .
\end{aligned}
$$

Because of continuity we know that

$$
\left\|Y_{t}^{x}-Y_{t}^{x_{m}}\right\|^{2} \underset{m \longrightarrow \infty}{\longrightarrow} 0
$$

for all $(t, \omega)$. So it follows from uniform integrability in connection with (2.19) that

$$
E\left[\left\|Y_{t}^{x}-Y_{t}^{x_{m}}\right\|^{2}\right] \underset{m \longrightarrow \infty}{\longrightarrow} 0
$$

for all $t$. Then using (2.18) and dominated convergence shows that

$$
I_{2}=I_{2}(m) \underset{m \longrightarrow \infty}{\longrightarrow} 0 .
$$

In summary, we see that $M=M(x)$ with the derivative of the flow replaced by $Y_{t}, 0 \leq$ $t \leq T$ is continuous in $x$ on $U$.

\section{Application: Stock price model with stochastic volatility}

In this Section we propose a model for stock prices $S_{t}^{x_{1}, x_{2}}, 0 \leq t \leq T$ with stochastic volatility $\sigma_{t}^{x_{2}}, 0 \leq t \leq T$ described by the following SDE

$$
\begin{aligned}
S_{t}^{x_{1}, x_{2}} & =x_{1}+\int_{0}^{t} \mu S_{u}^{x_{1}, x_{2}} d u+\int_{0}^{t} g\left(\sigma_{u}^{x_{2}}\right) S_{u}^{x_{1}, x_{2}} d W_{u} \\
\sigma_{t}^{x_{2}} & =x_{2}+\int_{0}^{t} b\left(u, \sigma_{u}^{x_{2}}\right) d u+B_{t}^{H}, x_{1}, x_{2} \in \mathbb{R}, 0 \leq t \leq T,
\end{aligned}
$$

where $W$. is a Wiener process, which is independent of a fractional Brownian motion $B^{H}$ with Hurst parameter $H<\frac{1}{2(d+2)}=\frac{1}{6}$, and where $\mu \in \mathbb{R}, b \in L_{\infty, \infty}^{1, \infty}$ and $g: \mathbb{R} \longrightarrow(\alpha, \infty)$ belongs to $C_{b}^{2}(\mathbb{R})$ for same $\alpha>0$. Let us also assume that $\Omega=\Omega_{1} \times \Omega_{2}$ for sample spaces $\Omega_{1}, \Omega_{2}$, on which $W$. and $B^{H}$ are defined, respectively.

For a moment, let us assume that $b \in C_{c}^{\infty}\left((0, T) \times \mathbb{R}^{d}\right)$. Then $X_{t}^{x}:=\left(S_{t}^{x_{1}, x_{2}}, \sigma_{t}^{x_{2}}\right)^{*}, x=$ $\left(x_{1}, x_{2}\right)$ is Malliavin differentiable with respect to $Z=\left(Z^{(1)}, Z^{(2)}\right)^{*}=\left(W, B^{H}\right)^{*}$ with Malliavin derivative $D=\left(D^{W}, D^{H}\right)^{*}$ and we get

$$
D_{s} X_{t}^{x}
$$




$$
\begin{aligned}
= & \int_{s}^{t}\left(\begin{array}{ll}
\mu & 0 \\
0 & b^{\prime}\left(u, \sigma_{u}^{x_{2}}\right)
\end{array}\right) D_{s} X_{u}^{x} d u \\
& +\left(\sum_{j=1}^{2} \int_{s}^{t} \sum_{l=1}^{2} \frac{\partial}{\partial x_{l}} a_{i j}\left(S_{u}^{x_{1}, x_{2}}, \sigma_{u}^{x_{2}}\right)\left(D_{s} X_{u}^{x}\right)_{r l} d Z_{u}^{(j)}\right)_{1 \leq i, r \leq 2} \\
& +\chi_{[0, t]}(s)\left(a_{i j}\left(S_{s}^{x_{1}, x_{2}}, \sigma_{s}^{x_{2}}\right)\right)_{1 \leq i, j \leq 2} \\
= & \int_{s}^{t}\left(\begin{array}{ll}
\mu & 0 \\
0 & b^{1}\left(u, \sigma_{u}^{x_{2}}\right)
\end{array}\right) D_{s} X_{u}^{x} d u \\
& +\left(\int_{s}^{t} \sum_{l=1}^{2} \frac{\partial}{\partial x_{l}} a_{i 1}\left(S_{u}^{x_{1}, x_{2}}, \sigma_{u}^{x_{2}}\right)\left(D_{s} X_{u}^{x}\right)_{r l} d W_{u}\right)_{1 \leq i, r \leq 2} \\
& +\chi_{[0, t]}(s)\left(a_{i j}\left(S_{s}^{x_{1}, x_{2}}, \sigma_{s}^{x_{2}}\right)\right)_{1 \leq i, j \leq 2}
\end{aligned}
$$

where

$$
\left(a_{i j}\left(x_{1}, x_{2}\right)\right)_{1 \leq i, j \leq 2}=\left(\begin{array}{ll}
g\left(x_{1}\right) x_{2} & 0 \\
0 & 1
\end{array}\right)
$$

We know that $X_{t}^{x, y}$ is twice continuously differentiable with respect to $(x, y)$. Then using a substitution formula for Wiener integrals [20, proof of Theorem 3.2.9], one finds similarly to the proof of Theorem 2.2 that

$$
D_{s} X_{t}^{x}=\frac{\partial}{\partial x} X_{t}^{s, X_{s}^{x}} \chi_{[0, t]}(s)\left(a_{i j}\left(S_{s}^{x_{1}, x_{2}}, \sigma_{s}^{x_{2}}\right)\right)_{1 \leq i, j \leq 2}
$$

Similarly, we get for a payoff function $\Phi \in C_{c}^{\infty}\left(\mathbb{R}^{2}\right)$ that

$$
\frac{\partial}{\partial x} E\left[\Phi\left(X_{T}^{x, n}\right)\right]=E\left[\Phi^{\prime}\left(X_{T}^{x}\right) \frac{\partial}{\partial x} X_{T}^{s, X_{s}^{x}} \frac{\partial}{\partial x} X_{s}^{x}\right]
$$

So

$$
\frac{\partial}{\partial x} E\left[\Phi\left(X_{T}^{x}\right)\right]=E\left[\Phi^{\prime}\left(X_{T}^{x}\right) D_{s} X_{T}^{x}\left(a_{i j}\left(S_{s}^{x_{1}, x_{2}}, \sigma_{s}^{x_{2}}\right)\right)_{1 \leq i, j \leq 2}^{-1} \frac{\partial}{\partial x} X_{s}^{x}\right]
$$

Hence, for a bounded measurable function $a$ summing up to one we obtain by means of the chain rule with respect to $D$. that

$$
\begin{aligned}
& \frac{\partial}{\partial x} E\left[\Phi\left(X_{T}^{x}\right)\right] \\
= & E\left[\int_{0}^{T}\left\{a(s) \Phi^{\prime}\left(X_{T}^{x}\right) D_{s} X_{T}^{x}\left(a_{i j}\left(S_{s}^{x_{1}, x_{2}}, \sigma_{s}^{x_{2}}\right)\right)_{1 \leq i, j \leq 2}^{-1} \frac{\partial}{\partial x} X_{s}^{x}\right\} d s\right] \\
= & E\left[\int_{0}^{T}\left\{a(s) D_{s} \Phi\left(X_{T}^{x}\right)\left(a_{i j}\left(S_{s}^{x_{1}, x_{2}}, \sigma_{s}^{x_{2}}\right)\right)_{1 \leq i, j \leq 2}^{-1} \frac{\partial}{\partial x} X_{s}^{x}\right\} d s\right]
\end{aligned}
$$

We have that

$$
\begin{aligned}
& \left(a_{i j}\left(S_{s}^{x_{1}, x_{2}}, \sigma_{s}^{x_{2}}\right)\right)_{1 \leq i, j \leq 2}^{-1} \frac{\partial}{\partial x} X_{s}^{x} \\
= & \left(\begin{array}{ll}
\left(S_{s}^{x_{1}, x_{2}} g\left(\sigma_{s}^{x_{2}}\right)\right)^{-1} \frac{\partial}{\partial x_{1}} S_{s}^{x_{1}, x_{2}} & \left(S_{s}^{x_{1}, x_{2}} g\left(\sigma_{s}^{x_{2}}\right)\right)^{-1} \frac{\partial}{\partial x_{2}} S_{s}^{x_{1}, x_{2}} \\
0 & \frac{\partial}{\partial x_{2}} \sigma_{s}^{x_{2}}
\end{array}\right) .
\end{aligned}
$$


Thus

$$
\begin{aligned}
& D_{s} \Phi\left(X_{T}^{x}\right)\left(a_{i j}\left(S_{s}^{x_{1}, x_{2}}, \sigma_{s}^{x_{2}}\right)\right)_{1 \leq i, j \leq 2}^{-1} \frac{\partial}{\partial x} X_{s}^{x} \\
= & \left(D_{s}^{W} \Phi\left(X_{T}^{x}\right)\left(S_{s}^{x_{1}, x_{2}} g\left(\sigma_{s}^{x_{2}}\right)\right)^{-1} \frac{\partial}{\partial x_{1}} S_{s}^{x_{1}, x_{2}},\right. \\
& D_{s}^{W} \Phi\left(X_{T}^{x}\right)\left(S_{s}^{x_{1}, x_{2}} g\left(\sigma_{s}^{x_{2}}\right)\right)^{-1} \frac{\partial}{\partial x_{2}} S_{s}^{x_{1}, x_{2}} \\
& \left.+D_{s}^{H} \Phi\left(X_{T}^{x}\right) \frac{\partial}{\partial x_{2}} \sigma_{s}^{x_{2}}\right)^{*} .
\end{aligned}
$$

So it follows that

$$
\begin{aligned}
& \frac{\partial}{\partial x} E\left[\Phi\left(X_{T}^{x}\right)\right] \\
= & \left(E\left[\int_{0}^{T} a(s) D_{s}^{W} \Phi\left(X_{T}^{x}\right)\left(S_{s}^{x_{1}, x_{2}} g\left(\sigma_{s}^{x_{2}}\right)\right)^{-1} \frac{\partial}{\partial x_{1}} S_{s}^{x_{1}, x_{2}} d s\right],\right. \\
& E\left[\int_{0}^{T} a(s) D_{s}^{W} \Phi\left(X_{T}^{x}\right)\left(S_{s}^{x_{1}, x_{2}} g\left(\sigma_{s}^{x_{2}}\right)\right)^{-1} \frac{\partial}{\partial x_{2}} S_{s}^{x_{1}, x_{2}} d s\right] \\
& \left.+E\left[\int_{0}^{T} a(s) D_{s}^{H} \Phi\left(X_{T}^{x}\right) \frac{\partial}{\partial x_{2}} \sigma_{s}^{x_{2}} d s\right]\right)^{*} .
\end{aligned}
$$

In fact, using the independence of $W$. and $B^{H}$, we can employ the proof of Theorem 2.2 and get that

$$
\begin{aligned}
& E\left[\int_{0}^{T} a(s) D_{s}^{H} \Phi\left(X_{T}^{x}\right) \frac{\partial}{\partial x_{2}} \sigma_{s}^{x_{2}} d s\right] \\
= & C_{H} E\left[\Phi\left(X_{T}^{x}\right) \int_{0}^{T} u^{-H-\frac{1}{2}} \int_{u}^{T} a(s-u)(s-u)^{\frac{1}{2}-H} s^{H-\frac{1}{2}} \frac{\partial}{\partial x} \sigma_{s-u}^{x_{2}} d B_{s} d u\right],
\end{aligned}
$$

where $B$. is a one-dimensional Brownian motion with respect to the representation of $B^{H}$ in $(2.2)$.

Finally, we can apply the duality formula with respect to $W$. and similar arguments as in the proof of Theorem 2.2 based on regular functions $g, b, \Phi$ and we obtain the following BEL-formula for our stock price model (3.1):

TheOREM 3.1. Let $U \subset \mathbb{R}^{2}$ be a bounded, open set and $b \in L_{\infty, \infty}^{1, \infty}$ in the stock price model (3.1). Further, assume that $g: \mathbb{R} \longrightarrow(\alpha, \infty)$ belongs to $C_{b}^{2}(\mathbb{R})$ for some $\alpha>0$ and that $\Phi: \mathbb{R}^{2} \longrightarrow \mathbb{R}$ satisfies

$$
\Phi\left(S_{\dot{T}}^{\cdot}, \sigma_{T}^{\cdot}\right) \in L^{2}(\Omega \times U, \mu \times d x) .
$$

In addition, let a be a bounded and measurable function on $[0, T]$, which sums up to 1 . Then

$$
\begin{aligned}
& \frac{\partial}{\partial x} E\left[\Phi\left(S_{T}^{x_{1}, x_{2}}, \sigma_{T}^{x_{2}}\right)\right] \\
= & \left(E\left[\Phi\left(X_{T}^{x}\right) \int_{0}^{T} a(s)\left(S_{s}^{x_{1}, x_{2}} g\left(\sigma_{s}^{x_{2}}\right)\right)^{-1} \frac{\partial}{\partial x_{1}} S_{s}^{x_{1}, x_{2}} d W_{s}\right],\right. \\
& E\left[\Phi\left(X_{T}^{x}\right) \int_{0}^{T} a(s)\left(S_{s}^{x_{1}, x_{2}} g\left(\sigma_{s}^{x_{2}}\right)\right)^{-1} \frac{\partial}{\partial x_{2}} S_{s}^{x_{1}, x_{2}} d W_{s}\right]
\end{aligned}
$$




$$
\left.+C_{H} E\left[\Phi\left(X_{T}^{x}\right) \int_{0}^{T} u^{-H-\frac{1}{2}} \int_{u}^{T} a(s-u)(s-u)^{\frac{1}{2}-H} s^{H-\frac{1}{2}} \frac{\partial}{\partial x} \sigma_{s-u}^{x_{2}} d B_{s} d u\right]\right)^{*}
$$

for almost all $x=\left(x_{1}, x_{2}\right) \in U$, where $C_{H}$ is a constant as given in Theorem 2.2.

REMARK 3.1. If $H<\frac{1}{2(d+3)}=\frac{1}{8}$, one can show just as in Theorem 2.2 that the right hand side of (3.2) has a continuous version.

\section{Appendix}

We start by stating some basic facts about fractional Brownian motion and then use these to recall some results on the Malliavin calculus with respect to fractional Brownian motion. For an in-depth treatment of this material see [20]. We end with a collection of some technical lemmas that we make use of in our paper.

4.1. Fractional Brownian motion Let $a, b \in \mathbb{R}$ with $a<b$. Let $f \in L^{p}([a, b])$ with $p \geq 1$ and $\alpha>0$. Introduce the left- and right-sided Riemann-Liouville fractional integrals as

$$
I_{a^{+}}^{\alpha} f(x)=\frac{1}{\Gamma(\alpha)} \int_{a}^{x}(x-y)^{\alpha-1} f(y) d y
$$

and

$$
I_{b^{-}}^{\alpha} f(x)=\frac{1}{\Gamma(\alpha)} \int_{x}^{b}(y-x)^{\alpha-1} f(y) d y
$$

for almost all $x \in[a, b]$, where $\Gamma$ is the Gamma function.

For a given integer $p \geq 1$, let $I_{a^{+}}^{\alpha}\left(L^{p}\right)\left(\right.$ resp. $\left.I_{b^{-}}^{\alpha}\left(L^{p}\right)\right)$ be the image of $L^{p}([a, b])$ of the operator $I_{a^{+}}^{\alpha}$ (resp. $I_{b^{-}}^{\alpha}$ ). If $f \in I_{a^{+}}^{\alpha}\left(L^{p}\right)$ (resp. $\left.f \in I_{b^{-}}^{\alpha}\left(L^{p}\right)\right)$ and $0<\alpha<1$ then we can define the left-and right-sided Riemann-Liouville fractional derivatives by

$$
D_{a^{+}}^{\alpha} f(x)=\frac{1}{\Gamma(1-\alpha)} \frac{d}{d x} \int_{a}^{x} \frac{f(y)}{(x-y)^{\alpha}} d y
$$

and

$$
D_{b^{-}}^{\alpha} f(x)=\frac{1}{\Gamma(1-\alpha)} \frac{d}{d x} \int_{x}^{b} \frac{f(y)}{(y-x)^{\alpha}} d y
$$

The left- and right-sided derivatives of $f$ can be also represented as

$$
D_{a^{+}}^{\alpha} f(x)=\frac{1}{\Gamma(1-\alpha)}\left(\frac{f(x)}{(x-a)^{\alpha}}+\alpha \int_{a}^{x} \frac{f(x)-f(y)}{(x-y)^{\alpha+1}} d y\right)
$$

and

$$
D_{b^{-}}^{\alpha} f(x)=\frac{1}{\Gamma(1-\alpha)}\left(\frac{f(x)}{(b-x)^{\alpha}}+\alpha \int_{x}^{b} \frac{f(x)-f(y)}{(y-x)^{\alpha+1}} d y\right)
$$

Using the above definitions, one obtains that

$$
I_{a^{+}}^{\alpha}\left(D_{a^{+}}^{\alpha} f\right)=f
$$


for all $f \in I_{a^{+}}^{\alpha}\left(L^{p}\right)$ and

$$
D_{a^{+}}^{\alpha}\left(I_{a^{+}}^{\alpha} f\right)=f
$$

for all $f \in L^{p}([a, b])$ and similarly for $I_{b^{-}}^{\alpha}$ and $D_{b^{-}}^{\alpha}$.

Let now $B^{H}=\left\{B_{t}^{H}, t \in[0, T]\right\}$ be a $d$-dimensional fractional Brownian motion with Hurst parameter $H \in(0,1 / 2)$, that is $B^{H}$ is a centered Gaussian process with a covariance function given by

$$
\left(R_{H}(t, s)\right)_{i, j}:=E\left[B_{t}^{H,(i)} B_{s}^{H,(j)}\right]=\delta_{i j} \frac{1}{2}\left(t^{2 H}+s^{2 H}-|t-s|^{2 H}\right), \quad i, j=1, \ldots, d,
$$

where $\delta_{i j}$ is one, if $i=j$, or zero else.

In the sequel we briefly recall the construction of the fractional Brownian motion, which can be found in [20]. For simplicity, consider the case $d=1$.

Let $\mathcal{E}$ be the set of step functions on $[0, T]$ and $\mathcal{H}$ be the Hilbert space given by the completion of $\mathcal{E}$ with respect to the inner product

$$
\left\langle 1_{[0, t]}, 1_{[0, s]}\right\rangle_{\mathcal{H}}=R_{H}(t, s) .
$$

From that we get an extension of the mapping $1_{[0, t]} \mapsto B_{t}$ to an isometry between $\mathcal{H}$ and a Gaussian subspace of $L^{2}(\Omega)$ with respect to $B^{H}$. We denote by $\varphi \mapsto B^{H}(\varphi)$ this isometry.

If $H<1 / 2$, one shows that the covariance function $R_{H}(t, s)$ has the representation

$$
R_{H}(t, s)=\int_{0}^{t \wedge s} K_{H}(t, u) K_{H}(s, u) d u
$$

where

$$
K_{H}(t, s)=c_{H}\left[\left(\frac{t}{s}\right)^{H-\frac{1}{2}}(t-s)^{H-\frac{1}{2}}+\left(\frac{1}{2}-H\right) s^{\frac{1}{2}-H} \int_{s}^{t} u^{H-\frac{3}{2}}(u-s)^{H-\frac{1}{2}} d u\right] .
$$

Here $c_{H}=\sqrt{\frac{2 H}{(1-2 H) \beta(1-2 H, H+1 / 2)}}$ and $\beta$ is the Beta function. See [20, Proposition 5.1.3].

Based on the kernel $K_{H}$, one can introduce by means (4.1) an isometry $K_{H}^{*}$ between $\mathcal{E}$ and $L^{2}([0, T])$ such that $\left(K_{H}^{*} 1_{[0, t]}\right)(s)=K_{H}(t, s) 1_{[0, t]}(s)$. This isometry has an extension to the Hilbert space $\mathcal{H}$, which has the following representations by means of fractional derivatives

$$
\left(K_{H}^{*} \varphi\right)(s)=c_{H} \Gamma\left(H+\frac{1}{2}\right) s^{\frac{1}{2}-H}\left(D_{T^{-}}^{\frac{1}{2}-H} u^{H-\frac{1}{2}} \varphi(u)\right)(s)
$$

and

$$
\begin{aligned}
\left(K_{H}^{*} \varphi\right)(s)= & c_{H} \Gamma\left(H+\frac{1}{2}\right)\left(D_{T^{-}}^{\frac{1}{2}-H} \varphi(s)\right)(s) \\
& +c_{H}\left(\frac{1}{2}-H\right) \int_{s}^{T} \varphi(t)(t-s)^{H-\frac{3}{2}}\left(1-\left(\frac{t}{s}\right)^{H-\frac{1}{2}}\right) d t .
\end{aligned}
$$


for $\varphi \in \mathcal{H}$. One also proves that $\mathcal{H}=I_{T^{-}}^{\frac{1}{2}-H}\left(L^{2}\right)$. See [11] and [1, Proposition 6].

Since $K_{H}^{*}$ is an isometry from $\mathcal{H}$ into $L^{2}([0, T])$, the $d$-dimensional process $W=$ $\left\{W_{t}, t \in[0, T]\right\}$ defined by

$$
W_{t}:=B^{H}\left(\left(K_{H}^{*}\right)^{-1}\left(1_{[0, t]}\right)\right)
$$

is a Wiener process and the process $B^{H}$ can be represented as

$$
B_{t}^{H}=\int_{0}^{t} K_{H}(t, s) d W_{s}
$$

See [1].

In what follows we also need the Definition of a fractional Brownian motion with respect to a filtration.

Definition 4.1. Let $\mathcal{G}=\left\{\mathcal{G}_{t}\right\}_{t \in[0, T]}$ be a filtration on $(\Omega, \mathcal{F}, P)$ satisfying the usual conditions. A fractional Brownian motion $B^{H}$ is called a $\mathcal{G}$-fractional Brownian motion if the process $W$ defined by (4.3) is a $\mathcal{G}$-Brownian motion.

In the following, let $W$ be a standard Wiener process on a filtered probability space $(\Omega, \mathfrak{A}, P),\left\{\mathcal{F}_{t}\right\}_{t \in[0, T]}$, where $\mathcal{F}=\left\{\mathcal{F}_{t}\right\}_{t \in[0, T]}$ is the natural filtration generated by $W$ and augmented by all $P$-null sets. Denote by $B^{H}$ the fractional Brownian motion with Hurst parameter $H \in(0,1 / 2)$ as in (4.4).

We aim at using a version of Girsanov's theorem for fractional Brownian motion which is due to [11, Theorem 4.9]. The version stated here corresponds to that in $[21$, Theorem 2]. To this end, we need the definition of an isomorphism $K_{H}$ from $L^{2}([0, T])$ onto $I_{0+}^{H+\frac{1}{2}}\left(L^{2}\right)$ with respec to the kernel $K_{H}(t, s)$ in terms of the fractional integrals as follows (see [11, Theorem 2.1]):

$$
\left(K_{H} \varphi\right)(s)=I_{0^{+}}^{2 H} s^{\frac{1}{2}-H} I_{0^{+}}^{\frac{1}{2}-H} s^{H-\frac{1}{2}} \varphi, \quad \varphi \in L^{2}([0, T]) .
$$

Using this and the properties of the Riemann-Liouville fractional integrals and derivatives, one can show that the inverse of $K_{H}$ can be represented as

$$
\left(K_{H}^{-1} \varphi\right)(s)=s^{\frac{1}{2}-H} D_{0^{+}}^{\frac{1}{2}-H} s^{H-\frac{1}{2}} D_{0^{+}}^{2 H} \varphi(s), \quad \varphi \in I_{0+}^{H+\frac{1}{2}}\left(L^{2}\right) .
$$

From this one obtains for absolutely continuous functions $\varphi$ (see [21]) that

$$
\left(K_{H}^{-1} \varphi\right)(s)=s^{H-\frac{1}{2}} I_{0^{+}}^{\frac{1}{2}-H} s^{\frac{1}{2}-H} \varphi^{\prime}(s) .
$$

TheOREM 4.1 (Girsanov's theorem for fBm). Let $u=\left\{u_{t}, t \in[0, T]\right\}$ be an $\mathcal{F}$-adapted process with integrable trajectories and set $\widetilde{B}_{t}^{H}=B_{t}^{H}+\int_{0}^{t} u_{s} d s, \quad t \in[0, T]$. Suppose that

(i) $\int_{0} u_{s} d s \in I_{0+}^{H+\frac{1}{2}}\left(L^{2}([0, T])\right), P$-a.s.

(ii) $E\left[\xi_{T}\right]=1$ where

$$
\xi_{T}:=\exp \left\{-\int_{0}^{T} K_{H}^{-1}\left(\int_{0} u_{r} d r\right)(s) d W_{s}-\frac{1}{2} \int_{0}^{T} K_{H}^{-1}\left(\int_{0} u_{r} d r\right)^{2}(s) d s\right\} .
$$

Then the shifted process $\widetilde{B}^{H}$ is an $\mathcal{F}$-fractional Brownian motion with Hurst parameter $H$ under the new probability $\widetilde{P}$ defined by $\frac{d \widetilde{P}}{d P}=\xi_{T}$.

REMARK 4.1. In the the multi-dimensional case, we define

$$
\left(K_{H} \varphi\right)(s):=\left(\left(K_{H} \varphi^{(1)}\right)(s), \ldots,\left(K_{H} \varphi^{(d)}\right)(s)\right)^{*}, \quad \varphi \in L^{2}\left([0, T] ; \mathbb{R}^{d}\right),
$$

where $*$ denotes transposition. Similarly for $K_{H}^{-1}$ and $K_{H}^{*}$. 


\subsection{Malliavin calculus}

Let $\mathcal{S}$ be the set of smooth and cylindrical random variables of the form

$$
F=f\left(B^{H}\left(\phi_{1}\right), \ldots, B^{H}\left(\phi_{n}\right)\right)
$$

where $n \geq 1, f \in C_{b}^{\infty}\left(\mathbb{R}^{n}\right)$ and $\phi_{1}, \ldots, \phi_{n} \in \mathcal{H}(\mathcal{H}$ is defined in the previous section). Given a random variable $F \in \mathcal{S}$ we define its derivative, as an element in $\mathcal{H}$, to be

$$
D^{H} F=\sum_{i=1}^{n} \frac{\partial f}{\partial x_{j}}\left(B^{H}\left(\phi_{1}\right), \ldots, B^{H}\left(\phi_{n}\right)\right) \phi_{j}
$$

For any $p \geq 1$, we define the Sobolev space $\mathbb{D}_{H}^{1, p}$ as the completion of $\mathcal{S}$ with respect to the norm

$$
\|F\|_{1, p}^{p}=\mathbb{E}|F|^{p}+\mathbb{E}\left\|D^{H} F\right\|_{\mathcal{H}}^{p}
$$

Note that that the previous holds for any $H \in(0,1)$ and in particular for $H=\frac{1}{2}$. Denote by $D:=D^{\frac{1}{2}}$ the Malliavin derivative with respect to $W$ and let $\mathbb{D}^{1, p}$ be its corresponding Sobolev space. We restate the following transfer principle, Proposition 5.2.1, [20], which links the $D$ and $D^{H}$.

Proposition 4.1. For any $F \in \mathbb{D}^{1, p}$

$$
K_{H}^{*} D^{H} F=D F
$$

A corollary of the previous is the following:

Lemma 4.1. Let $H \in(0,1)$ and $p>1$, then $B_{t}^{H}$ belongs to $\mathbb{D}^{1, p}$ for all $t>0$ and its Malliavin derivative is given by:

$$
D B_{t}^{H}(s)=\int_{0}^{\min (s, t)} K_{H}(t, u) d u
$$

and hence

$$
D_{\theta} B_{t}^{H}=K_{H}(t, \theta) I_{d}
$$

for any $\theta \in(0, t)$ and where $I_{d}$ is the identity matrix.

4.3. Technical lemmas In this article we also resort to the following technical lemma (see [5, Lemma 4.3]):

LEMMA 4.2. Let $\tilde{B}_{t}^{H}$ be a d-dimensional fractional Brownian motion with respect to $(\Omega, \mathfrak{A}, \tilde{P})$. Then for every $k \in \mathbb{R}$ we have

$$
\tilde{E}\left[\exp \left\{k \int_{0}^{T}\left|K_{H}^{-1}\left(\int_{0} b\left(r, \tilde{B}_{r}^{H}\right) d r\right)(s)\right|^{2} d s\right\}\right] \leq C_{H, d, \mu, T}\left(\|b\|_{L_{\infty}^{\infty}}\right)
$$

for some continuous increasing function $C_{H, d, k, T}$ depending only on $H, d, T$ and $k$.

In particular,

$$
\tilde{E}\left[\mathcal{E}\left(\int_{0}^{T} K_{H}^{-1}\left(\int_{0} b\left(r, \tilde{B}_{r}^{H}\right) d r\right)^{*}(s) d W_{s}\right)^{p}\right] \leq C_{H, d, \mu, T}\left(\|b\|_{L_{\infty}^{\infty}}\right),
$$


where $\mathcal{E}\left(M_{t}\right)$ is the Dolean-Dade exponential of a local martingale $M_{t}, 0 \leq t \leq T$ and where $\tilde{E}$ denotes expectation under $\tilde{P}$ and $*$ transposition.

In this paper, we will also make use of an integration by parts formula for iterated integrals based on shuffle permutations. For this purpose, let $m$ and $n$ be integers. Denote by $S(m, n)$ the set of shuffle permutations, i.e. the set of permutations $\sigma$ : $\{1, \ldots, m+n\} \rightarrow\{1, \ldots, m+n\}$ such that $\sigma(1)<\cdots<\sigma(m)$ and $\sigma(m+1)<\cdots<\sigma(m+n)$.

Introduce the $m$-dimensional simplex for $0 \leq \theta<t \leq T$,

$$
\Delta_{\theta, t}^{m}:=\left\{\left(s_{m}, \ldots, s_{1}\right) \in[0, T]^{m}: \theta<s_{m}<\cdots<s_{1}<t\right\} .
$$

The product of two simplices can be represented as follows

$$
\Delta_{\theta, t}^{m} \times \Delta_{\theta, t}^{n}=\bigcup_{\sigma \in S(m, n)}\left\{\left(w_{m+n}, \ldots, w_{1}\right) \in[0, T]^{m+n}: \theta<w_{\sigma(m+n)}<\cdots<w_{\sigma(1)}<t\right\} \cup \mathcal{N},
$$

where the set $\mathcal{N}$ has null Lebesgue measure. So, if $f_{i}:[0, T] \rightarrow \mathbb{R}, i=1, \ldots, m+n$ are integrable functions we get that

$$
\begin{aligned}
\int_{\Delta_{\theta, t}^{m}} \prod_{j=1}^{m} f_{j}\left(s_{j}\right) d s_{m} \ldots d s_{1} & \int_{\Delta_{\theta, t}^{n}} \prod_{j=m+1}^{m+n} f_{j}\left(s_{j}\right) d s_{m+n} \ldots d s_{m+1} \\
& =\sum_{\sigma \in S(m, n)} \int_{\Delta_{\theta, t}^{m+n}} \prod_{j=1}^{m+n} f_{\sigma(j)}\left(w_{j}\right) d w_{m+n} \cdots d w_{1} .
\end{aligned}
$$

A generalization of the latter relation is the following (see [5]):

Lemma 4.3. Let $n, p$ and $k$ be non-negative integers, $k \leq n$. Suppose we have integrable functions $f_{j}:[0, T] \rightarrow \mathbb{R}, j=1, \ldots, n$ and $g_{i}:[0, T] \rightarrow \mathbb{R}, i=1, \ldots, p$. We may then write

$$
\begin{aligned}
& \int_{\Delta_{\theta, t}^{n}} f_{1}\left(s_{1}\right) \ldots f_{k}\left(s_{k}\right) \int_{\Delta_{\theta, s_{k}}^{p}} g_{1}\left(r_{1}\right) \ldots g_{p}\left(r_{p}\right) d r_{p} \ldots d r_{1} f_{k+1}\left(s_{k+1}\right) \ldots f_{n}\left(s_{n}\right) d s_{n} \ldots d s_{1} \\
& =\sum_{\sigma \in A_{n, p}} \int_{\Delta_{\theta, t}^{n+p}} h_{1}^{\sigma}\left(w_{1}\right) \ldots h_{n+p}^{\sigma}\left(w_{n+p}\right) d w_{n+p} \ldots d w_{1}
\end{aligned}
$$

where $h_{l}^{\sigma} \in\left\{f_{j}, g_{i}: 1 \leq j \leq n, 1 \leq i \leq p\right\}$. Above $A_{n, p}$ stands for a subset of permutations of $\{1, \ldots, n+p\}$ such that $\# A_{n, p} \leq C^{n+p}$ for an appropriate constant $C \geq 1$. Here $s_{0}:=\theta$.

The proof of Lemma 2.4 relies on an important estimate (see e.g. Proposition 3.3 in [6] for the newer proof of this result). In order to state this result, we need some notation. Let $m$ be an integer and let $f:[0, T]^{m} \times\left(\mathbb{R}^{d}\right)^{m} \rightarrow \mathbb{R}$ be a function of the form

$$
f(s, z)=\prod_{j=1}^{m} f_{j}\left(s_{j}, z_{j}\right), \quad s=\left(s_{1}, \ldots, s_{m}\right) \in[0, T]^{m}, \quad z=\left(z_{1}, \ldots, z_{m}\right) \in\left(\mathbb{R}^{d}\right)^{m}
$$

where $f_{j}:[0, T] \times \mathbb{R}^{d} \rightarrow \mathbb{R}, j=1, \ldots, m$ are smooth functions with compact support. In addition, let $\varkappa:[0, T]^{m} \rightarrow \mathbb{R}$ be a function of the form

$$
\varkappa(s)=\prod_{j=1}^{m} \varkappa_{j}\left(s_{j}\right), \quad s \in[0, T]^{m}
$$

where $\varkappa_{j}:[0, T] \rightarrow \mathbb{R}, j=1, \ldots, m$ are integrable functions. 
Further, denote by $\alpha_{j}$ a multi-index and $D^{\alpha_{j}}$ its corresponding differential operator. For $\alpha=\left(\alpha_{1}, \ldots, \alpha_{m}\right)$ as an element of $\mathbb{N}_{0}^{d \times m}$ with $|\alpha|:=\sum_{j=1}^{m} \sum_{l=1}^{d} \alpha_{j}^{(l)}$, we write

$$
D^{\alpha} f(s, z)=\prod_{j=1}^{m} D^{\alpha_{j}} f_{j}\left(s_{j}, z_{j}\right)
$$

THEOREM 4.2. Let $B^{H}, H \in(0,1 / 2)$ be a standard $d$-dimensional fractional Brownian motion and functions $f$ and $\varkappa$ as in (4.6), respectively as in (4.7). Let $\theta, t \in[0, T]$ with $\theta<t$ and

$$
\varkappa_{j}(s)=\left(K_{H}(s, \theta)\right)^{\varepsilon_{j}}, \theta<s<t
$$

for every $j=1, \ldots, m$ with $\left(\varepsilon_{1}, \ldots, \varepsilon_{m}\right) \in\{0,1\}^{m}$. Let $\alpha \in\left(\mathbb{N}_{0}^{d}\right)^{m}$ be a multi-index. If

$$
H<\frac{\frac{1}{2}-\gamma}{\left(d-1+2 \sum_{l=1}^{d} \alpha_{j}^{(l)}\right)}
$$

for all $j$, where $\gamma \in(0, H)$ is sufficiently small, then there exists a universal constant $C$ (depending on $H, T$ and $d$, but independent of $m,\left\{f_{i}\right\}_{i=1, \ldots, m}$ and $\alpha$ ) such that for any $\theta, t \in[0, T]$ with $\theta<t$ we have

$$
\begin{aligned}
& \left|E \int_{\Delta_{\theta, t}^{m}}\left(\prod_{j=1}^{m} D^{\alpha_{j}} f_{j}\left(s_{j}, B_{s_{j}}^{H}\right) \varkappa_{j}\left(s_{j}\right)\right) d s\right| \\
\leq & C^{m+|\alpha|} \prod_{j=1}^{m}\left\|f_{j}\left(\cdot, z_{j}\right)\right\|_{L^{1}\left(\mathbb{R}^{d} ; L^{\infty}([0, T])\right)} \theta^{\left(H-\frac{1}{2}\right) \sum_{j=1}^{m} \varepsilon_{j}} \\
& \times \frac{\left(\prod_{l=1}^{d}\left(2\left|\alpha^{(l)}\right|\right) !\right)^{1 / 4}(t-\theta)^{-H(m d+2|\alpha|)-\left(H-\frac{1}{2}-\gamma\right) \sum_{j=1}^{m} \varepsilon_{j}+m}}{\Gamma\left(-H(2 m d+4|\alpha|)+2\left(H-\frac{1}{2}-\gamma\right) \sum_{j=1}^{m} \varepsilon_{j}+2 m\right)^{1 / 2}} .
\end{aligned}
$$

REMARK 4.2. The above theorem remains valid for time-homogeneous functions $\left\{f_{i}\right\}_{i=1, \ldots, m}$ in the Schwartz function space.

The proof of Lemma 2.4 also requires the following auxiliary result:

LEMMA 4.4. Let $n, p$ and $k$ be non-negative integers, $k \leq n$. Assume we have functions $f_{j}:[0, T] \rightarrow \mathbb{R}, j=1, \ldots, n$ and $g_{i}:[0, T] \rightarrow \mathbb{R}, i=1, \ldots, p$ such that

$$
f_{j} \in\left\{\frac{\partial^{\alpha_{j}^{(1)}+\ldots+\alpha_{j}^{(d)}}}{\partial^{\alpha_{j}^{(1)}} x_{1} \ldots \partial^{\alpha_{j}^{(d)}} x_{d}} b^{(r)}\left(u, X_{u}^{x}\right), r=1, \ldots, d\right\}, j=1, \ldots, n
$$

and

$$
g_{i} \in\left\{\frac{\partial^{\beta_{i}^{(1)}+\ldots+\beta_{i}^{(d)}}}{\partial^{\beta_{i}^{(1)}} x_{1} \ldots \partial^{\beta_{i}^{(d)}} x_{d}} b^{(r)}\left(u, X_{u}^{x}\right), r=1, \ldots, d\right\}, i=1, \ldots, p
$$

for $\alpha:=\left(\alpha_{j}^{(l)}\right) \in \mathbb{N}_{0}^{d \times n}$ and $\beta:=\left(\beta_{i}^{(l)}\right) \in \mathbb{N}_{0}^{d \times p}$, where $X^{x}$ is the strong solution to

$$
X_{t}^{x}=x+\int_{0}^{t} b\left(u, X_{u}^{x}\right) d u+B_{t}^{H}, 0 \leq t \leq T
$$


for $b=\left(b^{(1)}, \ldots, b^{(d)}\right)$ with $b^{(r)} \in C_{c}\left((0, T) \times \mathbb{R}^{d}\right)$ for all $r=1, \ldots, d$. So (as we shall say in the sequel) the product $g_{1}\left(r_{1}\right) \cdots \cdots g_{p}\left(r_{p}\right)$ has a total order of derivatives $|\beta|=$ $\sum_{l=1}^{d} \sum_{i=1}^{p} \beta_{i}^{(l)}$. We know from Lemma 4.3 that

$$
\begin{aligned}
& \int_{\Delta_{\theta, t}^{n}} f_{1}\left(s_{1}\right) \ldots f_{k}\left(s_{k}\right) \int_{\Delta_{\theta, s_{k}}^{p}} g_{1}\left(r_{1}\right) \ldots g_{p}\left(r_{p}\right) d r_{p} \ldots d r_{1} f_{k+1}\left(s_{k+1}\right) \ldots f_{n}\left(s_{n}\right) d s_{n} \ldots d s_{1} \\
& =\sum_{\sigma \in A_{n, p}} \int_{\Delta_{\theta, t}^{n+p}} h_{1}^{\sigma}\left(w_{1}\right) \ldots h_{n+p}^{\sigma}\left(w_{n+p}\right) d w_{n+p} \ldots d w_{1}
\end{aligned}
$$

where $h_{l}^{\sigma} \in\left\{f_{j}, g_{i}: 1 \leq j \leq n, 1 \leq i \leq p\right\}, A_{n, p}$ is a subset of permutations of $\{1, \ldots, n+$ $p\}$ such that $\# A_{n, p} \leq C^{n+p}$ for an appropriate constant $C \geq 1$, and $s_{0}=\theta$. Then the products

$$
h_{1}^{\sigma}\left(w_{1}\right) \cdots h_{n+p}^{\sigma}\left(w_{n+p}\right)
$$

have a total order of derivatives given by $|\alpha|+|\beta|$.

Proof. The result is proved by induction on $n$. For $n=1$ and $k=0$ the result is trivial. For $k=1$ we have

$$
\begin{aligned}
\int_{\theta}^{t} f_{1}\left(s_{1}\right) \int_{\Delta_{\theta, s_{1}}^{p}} g_{1}\left(r_{1}\right) \ldots g_{p}\left(r_{p}\right) & d r_{p} \ldots d r_{1} d s_{1} \\
= & \int_{\Delta_{\theta, t}^{p+1}} f_{1}\left(w_{1}\right) g_{1}\left(w_{2}\right) \ldots g_{p}\left(w_{p+1}\right) d w_{p+1} \ldots d w_{1}
\end{aligned}
$$

where we have put $w_{1}=s_{1}, w_{2}=r_{1}, \ldots, w_{p+1}=r_{p}$. Hence the total order of derivatives involved in the product of the last integral is given by $\sum_{l=1}^{d} \alpha_{1}^{(l)}+\sum_{l=1}^{d} \sum_{i=1}^{p} \beta_{i}^{(l)}=$ $|\alpha|+|\beta|$.

Assume the result holds for $n$ and let us show that this implies that the result is true for $n+1$. Either $k=0,1$ or $2 \leq k \leq n+1$. For $k=0$ the result is trivial. For $k=1$ we have

$$
\begin{aligned}
& \int_{\Delta_{\theta, t}^{n+1}} f_{1}\left(s_{1}\right) \int_{\Delta_{\theta, s_{1}}^{p}} g_{1}\left(r_{1}\right) \ldots g_{p}\left(r_{p}\right) d r_{p} \ldots d r_{1} f_{2}\left(s_{2}\right) \ldots f_{n+1}\left(s_{n+1}\right) d s_{n+1} \ldots d s_{1} \\
& \quad=\int_{\theta}^{t} f_{1}\left(s_{1}\right)\left(\int_{\Delta_{\theta, s_{1}}^{n}} \int_{\Delta_{\theta, s_{1}}^{p}} g_{1}\left(r_{1}\right) \ldots g_{p}\left(r_{p}\right) d r_{p} \ldots d r_{1} f_{2}\left(s_{2}\right) \ldots f_{n+1}\left(s_{n+1}\right) d s_{n+1} \ldots d s_{2}\right) d s_{1} .
\end{aligned}
$$

From (4.5) we observe by using the shuffle permutations that the latter inner double integral on diagonals can be written as a sum of integrals on diagonals of length $p+n$ with products having a total order of derivatives given by $\sum_{l=1} \sum_{j=2}^{n+1} \alpha_{j}^{(l)}+\sum_{l=1}^{d} \sum_{i=1}^{p} \beta_{i}^{(l)}$. Hence we obtain a sum of products, whose total order of derivatives is $\sum_{l=1}^{d} \sum_{j=2}^{n+1} \alpha_{j}^{(l)}+$ $\sum_{l=1}^{d} \sum_{i=1}^{p} \beta_{i}^{(l)}+\sum_{l=1}^{d} \alpha_{1}^{(l)}=|\alpha|+|\beta|$.

For $k \geq 2$ we have (in connection with Lemma 4.3) from the induction hypothesis that

$$
\begin{gathered}
\int_{\Delta_{\theta, t}^{n+1}} f_{1}\left(s_{1}\right) \ldots f_{k}\left(s_{k}\right) \int_{\Delta_{\theta, s_{k}}^{p}} g_{1}\left(r_{1}\right) \ldots g_{p}\left(r_{p}\right) d r_{p} \ldots d r_{1} f_{k+1}\left(s_{k+1}\right) \ldots f_{n+1}\left(s_{n+1}\right) d s_{n+1} \ldots d s_{1} \\
=\int_{\theta}^{t} f_{1}\left(s_{1}\right) \int_{\Delta_{\theta, s_{1}}^{n}} f_{2}\left(s_{2}\right) \ldots f_{k}\left(s_{k}\right) \int_{\Delta_{\theta, s_{k}}^{p}} g_{1}\left(r_{1}\right) \ldots g_{p}\left(r_{p}\right) d r_{p} \ldots d r_{1}
\end{gathered}
$$




$$
\begin{array}{r}
\times f_{k+1}\left(s_{k+1}\right) \ldots f_{n+1}\left(s_{n+1}\right) d s_{n+1} \ldots d s_{2} d s_{1} \\
=\sum_{\sigma \in A_{n, p}} \int_{\theta}^{t} f_{1}\left(s_{1}\right) \int_{\Delta_{\theta, s_{1}}^{n+p}} h_{1}^{\sigma}\left(w_{1}\right) \ldots h_{n+p}^{\sigma}\left(w_{n+p}\right) d w_{n+p} \ldots d w_{1} d s_{1},
\end{array}
$$

where each of the products $h_{1}^{\sigma}\left(w_{1}\right) \cdots \cdots h_{n+p}^{\sigma}\left(w_{n+p}\right)$ have a total order of derivatives given by $\sum_{l=1} \sum_{j=2}^{n+1} \alpha_{j}^{(l)}+\sum_{l=1}^{d} \sum_{i=1}^{p} \beta_{i}^{(l)}$. Thus we get a sum with respect to a set of permutations $A_{n+1, p}$ with products having a total order of derivatives which is

$$
\sum_{l=1}^{d} \sum_{j=2}^{n+1} \alpha_{j}^{(l)}+\sum_{l=1}^{d} \sum_{i=1}^{p} \beta_{i}^{(l)}+\sum_{l=1}^{d} \alpha_{1}^{(l)}=|\alpha|+|\beta| .
$$

\section{REFERENCES}

[1] Alòs, E., Mazet, O., Nualart, D., Stochastic calculus with respect to Gaussian processes. Annals of Probability 29, 766-801 (2001). 4.1, 4.1

[2] Amine, O., Baños, D., Proske, F.: Regularity properties of the stochastic flow of a skew fractional Brownian motion. arXiv:1805.04889v1 (2018). 1

[3] Baños, D., Duedahl, S., Meyer-Brandis, T., Proske, F.: Construction of Malliavin differentiable strong solutions of SDE's under an integrability conditionon the drift without the YamadaWatanabe principle. To appear in: Annales de II.H.P. Probabilites et statistiques (2018). 1

[4] Baños, D., Duedahl, S., Meyer-Brandis, T., Proske, F.: Computing deltas without derivatives. Finance Stoch.. ISSN 0949-2984. 21(2), p. 509-549 (2017). 1

[5] Baños, D., Nilssen, T., Proske, F.: Strong existence and higher order Fréchet differentiability of stochastic flows of fractional Brownian motion driven SDE's with singular drift. Journal of Dynamics and Differential Equations. (2019). 1, 2, 2, 2, 2, 2.2, 4.3, 4.3

[6] Baños, D., Ortiz-Latorre, S., Pilipenko, A., Proske, F.: Strong solutions of SDE's with generalized drift and multidimensional fractional Brownian initial noise. arXiv:1705.01616v2 [math.PR] (2018). 1, 2, 4.3

[7] Bismut, J.: Large Deviations and the Malliavin Calculus. Birkhäuser, Basel (1984). 1

[8] Elworthy, K.D., Li, X.-M.: Formulae for the derivatives of heat semigroups. Journal of Funct. Anal. 125, 252-286 (1994). 1

[9] Catellier, R., Gubinelli, M.: Averaging along irregular curves and regularisation of ODE's. Stoch. Proc. and their Appl., Vol. 126, Issue 8, p. 2323-2366 (August 2016). 2, 2.2

[10] Da Prato, G., Malliavin, P., Nualart, D.: Compact families of Wiener functionals. C. R. Acad. Sci. Paris, t. 315, Série I, 1287-1291 (1992). 2

[11] Decreusefond, L., Üstünel, A.S.: Stochastic analysis of the fractional Brownian motion. Potential Analysis 10, 177-214 (1998). 4.1, 4.1

[12] Di Nunno, Øksendal, B., Proske, F.: Malliavin Calculus for Lévy Processes with Applications to Finance. Springer (2008).

[13] Fan, X.-L., Ren, Y.: Bismut formulas and applications fro stochastic (functional) differential equations driven by fractional Brownian motions. Stoch. Dyn. 17, 1750028 (2017). 1

[14] Fournié, E., Lasry, J.-M., Lebuchoux, J., Lions, P.-L., Touzi, N.: Applications of Malliavin Calculus to Monte Carlo methods in finance. Finance Stoch., 3(4):391-412 (1999). 1, 1

[15] Fournié, E., Lasry, J.-M., Lebuchoux, J., Lions, P.-L.: Applications of Malliavin Calculus to Monte Carlo methods in finance. II. Finance Stoch., 5(2):201-236 (2001). 1

[16] Gatheral, Jim, Thibault Jaisson, and Mathieu Rosenbaum. "Volatility is rough." Quantitative Finance 18.6 (2018): 933-949. 1, 2.2

[17] Kunita, H.: Stochastic Flows and Stochastic Differential Equations. Cambridge Univ. Press (1988). 2, 2

[18] Lizorkin, P.I. Fractional integration and differentiation, Encyclopedia of Mathematics, Springer, (2001)

[19] Menoukeu-Pamen, O., Meyer-Brandis, T., Nilssen, T., Proske, F., Zhang, T.: A variational approach to the construction and Malliavin differentiability of strong solutions of SDE's. Math. Ann. 357 (2), 761-799, (2013). 1, 2 
[20] Nualart, D.: The Malliavin Calculus and Related Topics. 2nd edition, Spinger (2010). 2, 2, 3, 4, $4.1,4.1,4.2$

[21] Nualart, D., Ouknine, Y.: Regularization of differential equations by fractional noise. Stochastic Processes and their Applications,102(1), 103-116 (2002). 2, 4.1

[22] Samko, S. G., Kilbas, A. A., Marichev, O. L. Fractional Integrals and Derivatives. Theory and Applications, Gordon and Breach, (1993).

[23] Zhang, X.: Stochastic differential equations with Sobolev diffusion and singular drift and applications. Ann. Appl. Probab., Vol. 26, No. 5, 2697-2732 (2016). 